Western University Scholarship@Western

1995

\title{
Innovative vs. Imitative R\&D and Economic Growth
}

Jinli Zeng

Follow this and additional works at: https://ir.lib.uwo.ca/economicsresrpt

Part of the Economics Commons

Citation of this paper:

Zeng, Jinli. "Innovative vs. Imitative R\&D and Economic Growth." Department of Economics Research Reports, 9506. London, ON: Department of Economics, University of Western Ontario (1995). 


\title{
RESEARCH REPORT 9506
}

\section{Innovative vs. Imitative $R \& D$ and Economic Growth}

by

\section{Jinl1 Zens}

September 1994

\author{
Department of Economics \\ Social Sclence Centre \\ Un1versity of Western Ontario \\ London. Ontar10. CANADA \\ N6A $5 \mathrm{C2}$
}




\title{
INNOVATIVE VS. IMITATIVE R\&D AND ECONOMIC GROWTH
}

\author{
Jinli Zeng* \\ First Draft: November, 1993 \\ This Version: September, 1994
}

\begin{abstract}
The objective of this paper is to study the relationship between resource allocation and $R \& D$ on the one hand, and economic growth and welfare on the other. The paper presents a multi-sector dynamic general equilibrium growth model, in which resource allocation between innovative and imitative $R \& D$ is the key to the speed of economic growth. In this model, innovations and imitations can occur in the same sector at the same time and economic growth is driven by innovation through its interactions with imitation.

We discuss two types of imitations: rent-seeking imitations and productive imitations. We identify the channels through which innovation and imitation interact with each other. In the case where imitations are of the rent-seeking type, we show that subsidizing innovation is not necessarily equivalent to taxing imitation: while taxing imitative $R \& D$ always induces more investment in innovative $R \& D$ and less investment in imitative $R \& D$, subsidizing innovative $R \& D$ always encourages innovation but it discourages imitation only if the effective employment in innovative $R \& D$ is high enough relative to the effective employment in imitative $R \& D$; if the effective employment in innovative $R \& D$ is relatively low, then subsidizing innovation also attracts imitation. In the case where imitations are productive, we show that, in addition to the "nonequivalence" result, taxing imitative $R \& D$ may induce more imitations. In both cases, we show that a subsidy to innovative $R \& D$ always speeds up economic growth while a subsidy to imitative $R \& D$ always does the opposite, but the effects on welfare of both subsidies are ambiguous.
\end{abstract}

\footnotetext{
*Department of Economics, The University of Western Ontario, London, Ontario, Canada N6A 5C2. I would like to thank my advisor Peter Howitt for his guidance and Ake Blomqvist and Joel Fried for helpful suggestions and comments.
} 


\section{Introduction}

It has been recognized by more and more economists that technological progress is probably the most important source of economic growth. This can been seen from the literature on endogenous growth. All endogenous growth models base economic growth on technological progress but through different channels. For example, in the first endogenous growth model (Romer (1986)), technological progress was achieved along with physical capital accumulation; Lucas (1988) focused on technological progress through human capital accumulation; Aghion and Howitt (1992) and Grossman and Helpman (1991a) emphasized the importance of industrial innovations; and Dinopoulos (1991), Grossman and Helpman (1991b), Segerstrom (1991) and Davidson and Segerstrom (1993) (hereafter DGSD) considered two channels - innovations and imitations - at the same time. In this paper, we share with the DGSD the same belief that both innovation and imitation are essential to technological progress and thus to economic growth.

Innovation and imitation interact with each other in the process of technological progress. On the one hand, they encourage each other. Successful innovations open up new opportunities for imitations and therefore induce more resources to be spent in imitative $R \& D$, while imitations speed up the spread of the application of innovations. But on the other hand, they also discourage each other. Further innovations render the previous imitations obsolete and thus weaken the incentive for imitators to invest in imitative $R \& D$, and successful imitations increase the product market competition, therefore they discourage the innovators to invest in innovative R\&D. While the empirical evidence shows that like innovation, imitation is also an important economic phenomenon, the literature on $R \& D$ races pays very little attention to it. In this literature, imitation is either exogenously determined or totally ignored. This paper is an attempt along the line of the DGSD to further understand the interactions of 
innovation and imitation in the process of promoting technological progress, pushing economic growth and improving welfare, and to see how public policies can influence this process.

We adopt a dynamic general equilibrium framework similar to Segerstrom (1991) and Davidson and Segerstrom (1993), which are due to Grossman and Helpman (1991b). But following Aghion and Howitt (1991) (Appendix 2), we model the processes of innovation and imitation in such a way that innovations and imitations occur randomly and independently across firms, across sectors and over time. In this model, economic growth is driven by the interactions of innovation and imitation, but growth rate is determined by innovation only although both innovation and imitation (in the case where imitations are productive) contribute to welfare. We discuss two types of imitations - rent-seeking imitations and productive imitations. For both types of imitations, we identify the channels through which the investment (i.e. employment) ${ }^{1}$ in innovative and imitative $R \& D$ affects the values of innovation and imitation. In both cases, the employment in innovative $R \& D$ has a "business-stealing" effect on the values of innovation and imitation and a positive competition effect on the value of imitation; the employment in imitative R\&D has a negative competition effect on the values of both innovation and imitation. ${ }^{2}$ In the case where imitations are of the rent-seeking type, we show that there may exist three stationary equilibria depending on the values of the model's parameters. The first equilibrium involves zero investment in both innovative and imitative $R \& D$. As a result, there is no growth. In the second equilibrium, firms invest only in innovative $R \& D$. Therefore, the model degenerates to an innovation-driven growth model such as Aghion and Howitt (1992) and Grossman and Helpman (1991a). Our focus is on the third equilibrium, in which firms invest in both innovative and imitative $R \& D$. Several interesting results have been obtained. The most striking

\footnotetext{
${ }^{1}$ Throughout the paper, we use investment and employment interchangeably.

${ }^{2}$ All these effects are explained in section 2.
} 
one may be that subsidizing innovation and taxing imitation are not necessarily equivalent. The effects of these two policies on the investment in innovative $R \& D$ are the same: both of them induce more investment in innovative $R \& D$. But their effects on the investment in imitative $R \& D$ can be different. While taxing imitation always reduces investment in imitative $R \& D$, subsidizing innovation may or may not reduce investment in imitative $R \& D$ depending on the effective employment in innovative $R \& D$ relative to that in imitative $R \& D$ : if the employment in innovative $R \& D$ is relatively high, then, intuitively, subsidization of innovation discourages imitations; but if the effective employment in innovative $R \& D$ is relatively low, then the subsidization of innovation not only encourages innovation but also induces imitation.

In the case where imitations are productive, we identify two more channels through which the employment in innovative and imitative $R \& D$ affects the values of innovation and imitation. That is, in addition to those effects mentioned above, the employment in both innovative and imitative R\&D has a positive and negative dynamic competition effect on the values of both innovation and imitation. ${ }^{3}$ We focus on the equilibrium with both innovation and imitation. Numerical examples show that an increase in the subsidy to innovative $R \& D$ induces more employment in innovative $R \& D$, and it may increase or decrease the employment in imitative $R \& D$; an increase in the subsidy to imitative $R \& D$ reduces the employment in innovative $R \& D$, and similar to the subsidy to innovative $R \& D$, it may increase or decrease the employment in imitative R\&D.

In both cases, we show through numerical examples that a government subsidy to innovative or imitative $R \& D$ may or may not improve welfare.

\footnotetext{
${ }^{3}$ The positive and negative dynamic competition effects are explained in section 5 .
} 
Closely related to the present paper are the DGSD mentioned above. We adopt the similar basic framework: preferences are similar; production technologies are the same; and as in Dinopoulos (1991), imitations also take the form of producing new varieties. However, the processes of innovation and imitation are modelled in a different way. As in Aghion and Howitt (1991) (Appendix 2), we model innovations and imitations in such a way that innovations and imitations occur randomly and independently across firms, across sectors and over time. The DGSD assumes that both innovative and imitative $R \& D$ are targeted to specific sectors. In Grossman and Helpman (1991b), the static Bertrand competition is used to analyze the product markets and imitation is driven by factor price differences across countries, so innovations and imitations can not occur in the same country. In Dinopoulos (1991), Segerstrom (1991) and Davidson and Segerstrom (1993), imitation is assumed to be driven by sharing profit with the innovator (by producing different varieties to compete with the innovator in Dinopoulos (1991) and through collusion in Segerstrom (1991) and Davidson and Segerstrom (1993)), and equilibria are constructed such that at any point in time, some sectors are targeted by innovators and other sectors are targeted by imitators, therefore innovations and imitations can not occur in the same sector at the same time, although they can coexist in the same country. In this model, the way of modelling innovation and imitation leads to a different scenario: at any point in time, each sector has potential innovators and imitators; therefore innovations and imitations can occur in the same sector at the same time. We believe that this is consistent with casual observations.

The rest of this paper is organized as follows. Sections 2-4 are restricted to the case where imitation is of the rent-seeking type. The next section describes the economic environment and sets up the basic framework. Three stationary equilibria are discussed in section 3 . Section 4 focuses on the equilibrium with both innovation and imitation to analyze the effects of exogenous changes in the model's parameters and of the government policies. In section 
5, we consider productive imitations. We discuss the welfare properties of the laissez faire equilibrium in section 6 . Finally, some concluding remarks are given in section 7 .

\section{The Model}

The basic framework is similar to Segerstrom (1991) and Davidson and Segerstrom (1993) which are due to Grossman and Helpman (1991b). However, following Aghion and Howitt (1991) (Appendix 2), we model the processes of innovation and imitation in such a way that innovations and imitations occur randomly and independently across firms, across sectors and over time.

\subsection{Preferences}

The model economy consists of a continuum of sectors, indexed by $i$, located on $[0,1]$ and is populated with identical infinitely-lived individuals with measure $N$. The representative individual's intertemporal utility function is assumed to be given by

$$
U=\int_{0}^{\infty} e^{-\rho t} u(t) d t
$$

where $\rho$ is the individual's subjective discount rate, and $u(t)$ is the individual's instantaneous utility function which is assumed to take the following form

$$
u(t)=\int_{0}^{1} \ln \left(\sum_{\tau=0}^{q_{i}} \gamma^{\tau} Z_{i \tau}\right) d i
$$

where $\tau$ refers to vintage (quality) $\tau,{ }^{4} q_{i}$ is the number of successful innovations in sector $i$ up to time $t, \gamma>1$ is a measure of the size of innovation which represents the quality improvement of a new product relative to its old counterpart, and $Z_{i \tau}$ is a utility index for

\footnotetext{
${ }^{4} \mathrm{~A}$ product of vintage $\tau$ is a product whose quality has been improved $\tau$ times since time 0 .
} 
consumption of the products of vintage $\tau$ in sector $i$. We assume that this subutility function is

$$
Z_{i \tau}=\Omega\left(M_{i \tau}\right)\left(M_{i \tau} \prod_{j=1}^{M_{i \tau}} x_{i j \tau}^{\frac{1}{M_{i \tau}}}\right)
$$

where $x_{i j \tau}$ is the consumption of variety $j$ of vintage $\tau$ in sector $i, M_{i \tau}$ is the number of varieties of vintage $\tau$ in sector $i$, and $\Omega\left(M_{i \tau}\right)$ represents the individual's preference for varieties. The function $\Omega\left(M_{i \tau}\right)$ is assumed to have the following properties: (i) $\Omega^{\prime}\left(M_{i \tau}\right) \geq 0$, that is, the individual at least weakly prefer more varieties (if $\Omega^{\prime}\left(M_{i \tau}\right)=0$, then imitations are of the rent-seeking type because they do not contribute to welfare; if $\Omega^{\prime}\left(M_{i \tau}\right)>0$, then imitations are productive because they contribute to welfare.); (ii) $\lim _{M_{i \tau} \rightarrow \infty} \Omega\left(M_{i \tau}\right) \leq \gamma$, indicating that the individual's preference for varieties is not too strong (or equivalently, the quality improvements are large enough) so that new products can replace old ones; (iii) $\Omega(1)=1$, this is an assumption without loss of generality. In section 3 and section 4 , we consider the case where $\Omega\left(M_{i \tau}\right)=1$ for all $M_{i r}$ (therefore, $\Omega^{\prime}\left(M_{i \tau}\right)=0$ ). The case where $\Omega^{\prime}\left(M_{i \tau}\right)>0$ is left for section 5 . The functional form (3) is equivalent to assuming that the individuals' preferences over different varieties are highly diversified and the population is large so that by the law of large number each variety has the same demand if it has the same price. So (3) represents the "average" individual's preferences over varieties. The representative individual's budget constraint is

$$
\int_{0}^{\infty} e^{-R(t)} E(t) d t=W_{0}
$$

where $R(t)$ is the cumulative interest factor, $W_{0}$ is the discounted expected life time income at time 0 , and $E(t)$ the total expenditure at time $t$ which is given by

$$
E(t)=\int_{0}^{1} E_{i}(t) d i=\int_{0}^{1} \sum_{\tau=0}^{q_{i}} E_{i \tau}(t) d i=\int_{0}^{1}\left(\sum_{\tau=0}^{q_{i}} \sum_{j=1}^{M_{i \tau}} p_{i j \tau} x_{i j \tau}\right) d i,
$$

where $E_{i}(t)$ is the expenditure on the products of sector $i$ at time $t, E_{i \tau}(t)$ is the expenditure on the varieties of vintage $\tau$ in sector $i$ at time $t$, and $p_{i j \tau}$ is the price (in terms of labor) of 
variety $j$ of vintage $\tau$ in sector $i$.

Since the individual's preferences defined by (1), (2) and (3) exhibit separability across varieties, across vintages, across sectors and over time, the consumer's problem can be decomposed into four sub-problems: First, given the expenditure on the varieties of vintage $\tau$ in sector $i, E_{i \tau}(t)$, the consumer chooses the quantity of each variety, $x_{i j \tau}$, to maximize (3) subject to the constraint, $\sum_{j=1}^{M_{i \tau}} p_{i j \tau} x_{i j \tau}=E_{i \tau}$. The first-order conditions for this maximization problem give the demand functions ${ }^{5}$

$$
x_{i j \tau}=\frac{E_{i \tau}}{M_{i \tau} p_{i j \tau}} .
$$

Then the indirect utility function associated with vintage $\tau$ of sector $i$ is

$$
Z_{i \tau}=\Delta_{\tau} E_{i \tau}
$$

where $\Delta_{\tau} \equiv \Omega\left(M_{i \tau}\right)\left(\prod_{j=1}^{M_{i \tau}} \frac{-\frac{1}{M_{i j \tau}}}{M_{i \tau}}\right)$. Second, given the expenditure on the products of sector $i$, $E_{i}$, the consumer allocates it among vintages within sector $i$. That is, the consumer chooses the expenditure on each vintage, $E_{i \tau}$, to maximize

$$
u_{i} \equiv \ln \left(\sum_{\tau=0}^{q_{i}} \gamma^{\tau} \Delta_{\tau} E_{i \tau}\right)
$$

subject to $\sum_{\tau=0}^{q_{i}} E_{i \tau}=E_{i}$. Since products of different vintages adjusted for quality are perfect substitutes, the consumer chooses the vintage with the highest marginal utility of expenditure. It is easily verified that if the (product replacement) condition: ${ }^{6} \Delta_{\tau} \geq \Delta_{\tau-1}$, i.e.

$$
\gamma \Omega\left(M_{i \tau}\right)\left(\prod_{j=1}^{M_{i \tau}} p_{i j \tau}^{-\frac{1}{M_{i \tau}}}\right) \geq \Omega\left(M_{i(\tau-1)}\right)\left(\prod_{j=1}^{M_{i(\tau-1)}} p_{i j(\tau-1)}^{-\frac{1}{M_{i(\tau-1)}}}\right)
$$

\footnotetext{
${ }^{5}$ Notice that since the demand functions (6) exhibit a constant price elasticity and unitary expenditure elasticity, they can be aggregated across consumers to obtain aggregate demand functions with exactly the same form with $E_{i \tau}$ being the aggregate expenditure on the varieties of vintage $\tau$ in sector $i$ and correspondingly $E_{i}$ and $E$ being the aggregate expenditure on the products of sector $i$ and the aggregate total aggregate expenditure respectively. So in what follows, we take (6) as aggregate demand functions.

${ }^{6}$ This product replacement condition is similar to the one in Dinopoulos (1991).
} 
holds, then the consumer chooses vintage $\tau .^{7}$ Assume that once the $\tau$ th innovation succeeds, the $(\tau-1)$ th technology becomes common knowledge, then the prices of the varieties of vintage $(\tau-1)$ are driven to 1 (the marginal cost) when the products of vintage $\tau$ become available. We also assume that firms producing different varieties of the same vintage engage in price competition, so they charge the same price $p_{i \tau}$ (i.e. $p_{i j \tau}=p_{i \tau}$ ). Then the condition (9) becomes

$$
\frac{\gamma \Omega\left(M_{i \tau}\right)}{p_{i \tau}} \geq \Omega\left(M_{i(\tau-1)}\right)
$$

The price $p_{i \tau}$ will be set to satisfy this condition. Then the consumer will always just consume the-state-of-the-art products. Consequently, the indirect utility function associated with sector $i$ is given by

$$
u_{i}=\ln \left(\gamma^{q_{i}} \Delta_{q_{i}} E_{i}\right)
$$

and, correspondingly, the indirect instantaneous utility function is

$$
u(t)=\int_{0}^{1} \ln \left(\gamma^{q_{i}} \Delta_{q_{i}} E_{i}\right) d i .
$$

Third, the consumer allocates the given total expenditure $E(t)$ among the products of different sectors to maximize the instantaneous utility (12). Obviously, we have $E_{i}(t)=E(t)$. Then the consumer's life time utility is

$$
U=\int_{0}^{\infty} e^{-\rho t}\left\{\left[\int_{0}^{1} \ln \left(\gamma^{q_{i}} \Delta_{q_{i}}\right) d i\right]+\ln E\right\} d t
$$

Finally, the consumer chooses the time path of the total expenditure $E(t)$ to maximize his life time utility (13) subject to the budget constraint (4), which gives rise to

$$
\frac{\dot{E}}{E}=r(t)-\rho
$$

\footnotetext{
${ }^{7}$ We assume that even the equality holds (then the consumer is indifferent between the products of two vintages), the consumer still consumes only the higher quality vintage.
} 
where $\dot{E}$ denotes the time change rate of the total expenditure $E(t)$ and $\mathbf{r}(\mathrm{t})$ is the interest rate at time t. We intend to focus on stationary equilibria, ${ }^{8}$ so in what follows, all variables' time subscripts will be dropped. Also, the subscripts for varieties, vintages and sectors can be omitted because of the symmetrical structures of preferences and production technologies. ${ }^{9}$ This completes the description of the consumer's preferences. Now we turn to technologies and firms' problems.

\subsection{Technologies}

There are three types of productive activities: consumption good production, innovative R\&D and imitative R\&D. We assume that all these activities require only one input labour. It is also assumed that each individual is endowed with one unit of labour which is inelastically supplied to one of the above mentioned activities. So the total labour supply is $N$. Each type of activities is described as follows.

\section{Consumption Good Production}

We assume that consumption good producers in all sectors have access to the same constant-returns-to-scale technology with each unit of labour producing one unit of output regardless of quality and variety. But only successful innovators and imitators are able to produce the state-of-the-art products. Since the consumer buys only those products with the lowest quality-adjusted prices ( if lower-quality and higher-quality products have the same quality-adjusted price, then we have assumed that the consumer buys only higher-quality products), for each variety, with the assumptions that $\Omega(1)=1$ and $\Omega^{\prime}(M)=0$, the highest price the producer can charge is $p=\gamma$, where labor is taken as numeraire. From the demand

\footnotetext{
${ }^{8}$ In a stationary equilibrium, the total expenditure $E$ is constant, so the interest rate $r(t)$ must be constant and equal to the individual's subjective discount rate $\rho$.

${ }^{9}$ The production technologies will be described in the next subsection.
} 
functions (6), we know that the profit flow, $\pi(M)$, for each producer in any sector is

$$
\pi(M)=\left(\frac{\gamma-1}{\gamma}\right) \frac{E}{M}
$$

Note that, given the size of innovation and the consumer's expenditure, the profit flow depends only on the number of producers in that sector.

\section{Innovative $\mathbf{R} \& D$}

New higher-quality products have to be discovered through innovative $R \& D$ before they can be produced. Innovation is assumed to follow the Poisson process. The arrival rate depends on the productivity of innovative $\mathrm{R} \& \mathrm{D}, \lambda_{I}$, and the amount of labour employed, $y_{I}$. The technology for innovative $R \& D$ is assumed to be constant returns to scale. So the arrival rate is simply $\lambda_{I} y_{I}$. It is also assumed that innovations can not be targeted to specific sectors; they occur randomly and independently across firms, across sectors and over time. Once an innovative $R \& D$ firm succeeds in discovering a higher quality product in a certain sector, it becomes the sole producer of that sector and enjoys the monopoly profit until either another firm discovers an even higher quality product in the same sector at which time it is driven out of business or until some other imitative R\&D firms copy the state-of-the-art product to produce different varieties at which time it has to share the product market with these imitators. Let $W_{I}$ be the value of an innovative $\mathrm{R} \& \mathrm{D}$ firm and $V_{I}$ be the value of a successful innovation, then we have the Bellman equation

$$
\rho W_{I}=\max _{y_{I}>0}\left\{\lambda_{I} y_{I} V_{I}-y_{I}\right\}
$$

Assuming free entry into innovative $\mathrm{R} \& \mathrm{D}$, we have $W_{I}=0$. Then

$$
0=\max _{y_{I}>0}\left\{\lambda_{I} y_{I} V_{I}-y_{I}\right\}
$$

The first-order condition is

$$
\lambda_{I} V_{I} \leq 1, y_{I} \geq 0, \text { with at least one equality. }
$$


The value of innovation is given $b^{10}$

$$
V_{I}=\left[\frac{1}{\lambda_{C} n_{C}} \ln \left(1+\frac{\lambda_{C} n_{C}}{\rho+\lambda_{I} n_{I}}\right)\right]\left(\frac{\gamma-1}{\gamma}\right) E
$$

where $\lambda_{C}$ is the productivity parameter for an imitative $\mathrm{R} \& \mathrm{D}$ firm, $n_{I}$ and $n_{C}$ are the aggregate labour employment in innovative $\mathrm{R} \& \mathrm{D}$ and imitative $\mathrm{R} \& \mathrm{D}$ respectively. Here, $\lambda_{C}>\lambda_{I}$ is assumed to reflect the fact that imitation is easier than innovation. ${ }^{11}$

Notice that both the employment $n_{I}$ in innovative R\&D and the employment $n_{C}$ in imitative $R \& D$ have a negative effect on the value of innovation (see the signs of $\frac{\partial V_{I}}{\partial n_{I}}$ and $\frac{\partial V_{I}}{\partial n_{C}}$ in Appendix 3). That is, more employment in either type of research makes a successful innovation less valuable. The reasons for this are simple: an increase in the employment in innovative $R \& D$ shortens the length of time in which the previous innovator can enjoy its monopoly profit (the "business-stealing" effect); and an increase in the employment in imitative $R \& D$ reduces the innovator's profit flow by increasing the product market competition (the negative competition effect).

\section{Imitative $\mathbf{R} \& \mathbf{D}$}

As mentioned above, a successful innovator can not enjoy the monopoly profit forever. Other R\&D firms can engage in copying the state-of-the-art products to produce other varieties. Like innovation, imitation is also assumed to follow the Poisson process and occur randomly and independently across firms, across sectors and over time. The process of imitation has the same structure as that of innovation. By employing $y_{C}$ units of labour, an imitative $R \& D$ firm is successful in imitating the state-of-the-art product to produce a new variety with an instantaneous probability $\lambda_{C} y_{C}$. That is, the arrival rate of this Poisson process is $\lambda_{C} y_{C}$. In each sector, a successful imitator becomes the sole producer of the new

\footnotetext{
${ }^{10}$ See Appendix 2 for derivation

${ }^{11}$ Mansfield et al (1981) found that the ratio of imitation time to innovation time was about $\frac{2}{3}$.
} 
variety but shares the product market of that sector with the innovator and other imitators, if any. The processes of innovation and imitation generate a stationary distribution of the type $K$ of sector across sectors. ${ }^{12}$ At any point in time, some sectors have one producer (i.e. the innovator), some sectors have two (the innovator and one imitator), some have three (the innovator and two imitators) and so on. The value of a successful imitation depends crucially on this distribution. Let $W_{C}$ be the value of an imitative $R \& D$ firm and $V_{C}$ be the value of a successful imitation, and assume free entry into imitative $R \& D$, then we have a Bellman equation and a zero profit condition similar to (16) and (17). The first-order condition is:

$$
\lambda_{C} V_{C} \leq 1, y_{C} \geq 0, \text { with at least one equality, }
$$

where the value of imitation is given by ${ }^{13}$

$$
\begin{aligned}
V_{C}= & \frac{\lambda_{I} n_{I}}{\rho \lambda_{C} n_{C}}\left[\left(1+\frac{\lambda_{I} n_{I}}{\lambda_{C} n_{C}}\right) \ln \left(1+\frac{\lambda_{C} n_{C}}{\lambda_{I} n_{I}}\right)\right. \\
& \left.-\left(1+\frac{\rho+\lambda_{I} n_{I}}{\lambda_{C} n_{C}}\right) \ln \left(1+\frac{\lambda_{C} n_{C}}{\rho+\lambda_{I} n_{I}}\right)\right]\left(\frac{\gamma-1}{\gamma}\right) E .
\end{aligned}
$$

As has been shown in Appendix 4, higher employment $n_{C}$ in imitative $\mathrm{R} \& \mathrm{D}$ is associated with a lower value of imitation because the negative competition effect implies that higher employment in imitative R\&D leads to stronger competition in the product markets. However, the effect of an increase in the employment in innovative R\&D on the value of imitation depends on the effective employment $\lambda_{I} n_{I}$ in innovative $R \& D$ relative to the effective employment $\lambda_{C} n_{C}$ in imitative $R \& D$ : if the effective employment in innovative $R \& D$ is relatively low, then an increase in the employment in innovative $R \& D$ raises the value of imitation; if the effective employment in innovative $R \& D$ is relatively high, then a further increase in the employment in innovative $R \& D$ lowers the value of imitation. This is because an increase in

\footnotetext{
${ }^{12}$ The type of sector refers to the number of producers (also the number of varieties) in that sector. The type of sector (more precisely, the variable $K-1$ ) is geometrically distributed across sectors. See Appendix 1 for derivation.

${ }^{13}$ See Appendix 2 for derivation.
} 
Table 1: $\quad$ The Effects of Changes in $n_{I}$ and $n_{C}$ with $\Omega^{\prime}(M)=0$

\begin{tabular}{cll}
\hline \hline Employ. Increase & Effect on $V_{I}$ & Effect on $V_{C}$ \\
\hline$n_{I}$ & "Business-stealing" effect & "Business-stealing" effect \\
& $\Rightarrow V_{I}$ decreases & $\Rightarrow V_{C}$ decreases \\
& & Positive competition effect \\
& $\Rightarrow V_{C}$ increases \\
\hline$n_{C}$ & Negative competition effect & Negative competition effect \\
& $\Rightarrow V_{I}$ decreases & $\Rightarrow V_{C}$ decreases \\
\hline
\end{tabular}

Note: In addition to these effects, increases in $n_{I}$ and $n_{C}$ also decrease $V_{I}$ and $V_{C}$ by reducing the employment in consumption good production and thus making each producer's profit flow smaller.

the employment in innovative $R \& D$ has two offsetting effects. On the one hand, the increase in the employment in innovative $R \& D$ increases the probability with which an imitator can succeed in sectors with single producers (the positive competition effect), and therefore increase the profitability of imitation. But on the other hand, the increase in the employment in innovative $R \& D$ in the next period also shortens the length of time in which the imitator can enjoy the profit from producing a new variety (the "business-stealing" effect). So the net effect depends on the relative strength of these two forces. When the effective employment in innovative $R \& D$ is relatively low, the positive competition effect dominates the "businessstealing" effect, as a result, the value of imitation rises; when the effective employment in innovative R\&D is relatively high, the "business-stealing" effect dominates, therefore the value of imitation decreases.

The effects on the values of innovation and imitation of changes in the employment in innovative and imitative R\&D are shown in Figures 1 and 2 and summarized in Table 1 for future reference.

\section{Labour Market}

Assume full employment, we have the labour market clearing condition 


$$
n_{I}+n_{C}+\frac{E}{\gamma}=N
$$

where $E / \gamma$ is the total employment in consumption good production.

\section{Capital Market}

Finally, we assume there exists a perfect capital market. R\&D firms borrow funds from this market to pay their researchers and issue risky securities. The equilibrium interest rate $r$ clears the market at each moment in time. Since there is a continuum of sectors and innovations and imitations occur independently across firms and across sectors, individual investors are able to completely diversify away risk by holding a diversified portfolio of securities.

\section{$3 \quad$ Stationary Equilibria}

We consider only stationary equilibria. In equilibrium, the consumer's total expenditure $E$, the employment $n_{I}$ in innovative $\mathrm{R} \& \mathrm{D}$ and the employment $n_{C}$ in imitative $\mathrm{R} \& \mathrm{D}$ are all constant; the instantaneous interest rate $r$ is also constant and is equal to the individual's subjective discount rate $\rho$. A stationary equilibrium is described by a constant sequence of $\left\{n_{I}, n_{C}, E\right\}$ satisfying the following conditions

$$
\begin{aligned}
& {\left[\frac{\lambda_{I}}{\lambda_{C} n_{C}} \ln \left(1+\frac{\lambda_{C} n_{C}}{\rho+\lambda_{I} n_{I}}\right)\right]\left(\frac{\gamma-1}{\gamma}\right) E \leq 1, n_{I} \geq 0, \text { with at least one equality, }} \\
& \frac{\lambda_{I} n_{I}}{\rho n_{C}}\left[\left(1+\frac{\lambda_{I} n_{I}}{\lambda_{C} n_{C}}\right) \ln \left(1+\frac{\lambda_{C} n_{C}}{\lambda_{I} n_{I}}\right)-\left(1+\frac{\rho+\lambda_{I} n_{I}}{\lambda_{C} n_{C}}\right)\right. \\
& \left.\quad \ln \left(1+\frac{\lambda_{C} n_{C}}{\rho+\lambda_{I} n_{I}}\right)\right]\left(\frac{\gamma-1}{\gamma}\right) E \leq 1, n_{C} \geq 0, \text { with at least one equality, } \\
& n_{I}+n_{C}+\frac{E}{\gamma}=N .
\end{aligned}
$$


Table 2: $\quad$ Three Equilibria $\left(\Omega^{\prime}(M)=0\right)$

\begin{tabular}{cll}
\hline \hline $\begin{array}{c}\text { Relative speed } \lambda \\
\text { Labor endowment } N\end{array}$ & $(0,1 / 2)$ & {$[1 / 2, \infty)$} \\
\hline$\left(0, \frac{\rho}{\lambda_{I}(\gamma-1)}\right]$ & $n_{I}=n_{C}=0$ & $n_{I}=n_{C}=0$ \\
$\left(\frac{\rho}{\lambda_{I}(\gamma-1)}, \infty\right)$ & $n_{I}>0, n_{C}>0$ & $n_{I}>0, n_{C}=0$ \\
\hline
\end{tabular}

There exist three possible equilibria depending on the size of labor endowment and the productivity of innovative $R \& D$ relative to that of imitative $R \& D$ (see Table 2). Now we discuss the conditions for the existence of each equilibrium and the properties of each equilibrium.

\subsection{Zero R\&D Equilibrium}

There are two cases in which this equilibrium exists. The first case is that the labor endowment is too small. The second case is a limiting case where imitations are instantaneous. More specifically, letting $\lambda=\lambda_{I} / \lambda_{C}$, we have

Proposition 1: A zero R\&D equilibrium exists if and only if the labor endowment is too small, i.e., $N \leq \frac{\rho}{\lambda_{I}(\gamma-1)}$, and/ or imitations are instantaneous, i.e., $\lambda=0$.

The proof is given in Appendix 5. The intuitions behind this proposition are as follows.

In the first case, the labor endowment (correspondingly, the total expenditure) is so small that even without imitation, the profit an innovator can make will be too low to cover the cost of R\&D. So there is no innovative $R \& D$ and therefore there is no imitative $R \& D .{ }^{14}$ In the second case, imitations are so easy that once an innovation succeeds, an infinitely

\footnotetext{
${ }^{14}$ As has been shown in Appendix 5 , if $n_{I}=0$, then $V_{C}=0$.
} 
number of successful imitations follow immediately. Threatened by immediate imitations and thus zero returns to investment, no firms invest in innovative $R \& D$. With no investment in innovative $R \& D$ and thus no innovation, firms do not invest in imitative $R \& D$ either.

\subsection{Equilibrium with Innovation only}

If the labor endowment is large enough, but imitation is too difficult relative to innovation, then firms invest in innovative $R \& D$ but do not invest in imitative $R \& D$. Therefore we have the following proposition.

Proposition 2: An equilibrium with innovation only exists if and only if the labor endowment is large enough, i.e., $N>\frac{p}{\lambda_{I}(\gamma-1)}$, but imitation is too difficult, i.e., $\lambda \geq \frac{1}{2}$.

We prove this proposition in Appendix 6. The intuition is straightforward. If the labor endowment is large enough and innovation is not too difficult relative to imitation, then the profit potential is high enough to attract investment into innovative $R \& D$. But since imitation is not easy enough relative to innovation, the expected benefit will not be enough to cover the cost of investment in imitative $R \& D$. Therefore, in equilibrium, only innovative $R \& D$ occurs.

With zero employment in imitative $\mathrm{R} \& \mathrm{D}$, the value of a successful innovation is $V_{I}=$ $\frac{(\gamma-1)\left(N-n_{I}\right)}{\rho+\lambda_{I} n_{I}} .{ }^{15}$ The zero profit condition for an $\mathrm{R} \& \mathrm{D}$ firm $\lambda_{I} V_{I}=1$ implies $n_{I}=\left(\frac{\gamma-1}{\gamma}\right) N-\frac{\rho}{\lambda_{I} \gamma}$. This is exactly the result obtained in those endogenous growth models without imitation such as Aghion and Howitt (1992) and Grossman and Helpman (1991a). In equilibrium, the effects of changes in parameters are stated as follows.

\footnotetext{
${ }^{15} \lim _{n_{C} \rightarrow 0} V_{I}=\frac{(\gamma-1)\left(N-n_{I}\right)}{\rho+\lambda_{I} n_{I}}$
} 
Proposition 3: In the equilibrium with innovation only, the employment $n_{I}$ in innovative $R \& D$ increases with (i) an increase in the arrival rate parameter $\lambda_{I}$; (ii) an increase in the size $\gamma$ of innovation; (iii) an increase in labor endowment $N$ and (iv) a decrease in the individual's subjective discount rate $\rho$. The economic intuitions are explained in Aghion and Howitt (1992) pp. 334-335.

\subsection{Equilibrium with Both Innovation and Imitation}

If we rule out the limiting case (i.e. $\lambda=0$ ), then if the labor endowment is large enough, i.e., $N>\frac{\rho}{\lambda_{I}(\gamma-1)}$, and imitation is not too difficult, i.e., $\lambda<\frac{1}{2}$, then both innovative and imitative $R \& D$ occurs and returns to the investment in both types of $R \& D$ are equal.

In the rest of this section and the next section, we will focus on the equilibrium with both innovation and imitation. Solving (25) for $E$ and substituting it into (23) and (24) (with equalities) gives

$$
\begin{aligned}
& \frac{1}{\lambda_{C}} \ln \left(1+\frac{\lambda_{C} n_{C}}{\rho+\lambda_{I} n_{I}}\right) \\
& \quad=\frac{n_{I}}{\rho}\left[\left(1+\frac{\lambda_{I} n_{I}}{\lambda_{C} n_{C}}\right) \ln \left(1+\frac{\lambda_{C} n_{C}}{\lambda_{I} n_{I}}\right)-\left(1+\frac{\rho+\lambda_{I} n_{I}}{\lambda_{C} n_{C}}\right) \ln \left(1+\frac{\lambda_{C} n_{C}}{\rho+\lambda_{I} n_{I}}\right)\right], \\
& {\left[\frac{\lambda_{I}}{\lambda_{C} n_{C}} \ln \left(1+\frac{\lambda_{C} n_{C}}{\rho+\lambda_{I} n_{I}}\right)\right](\gamma-1)\left(N-n_{I}-n_{C}\right)=1 .}
\end{aligned}
$$

The first equation (26) is an equal profitability condition, which says that in equilibrium the expected returns to investment in innovative and imitative $R \& D$ are equal. We show in Appendix 8 that the employment $n_{I}$ in innovative $R \& D$ and the employment $n_{C}$ in imitative $R \& D$ described by this condition are positively related. That is, more employment in imitative $R \& D$ accompanies more employment in innovative $R \& D$ in order for this condition 
to hold.

The intuition behind this can be understood from the effects of changes in the employment in innovative and imitative $R \& D$ on the values of innovation and imitation. Let us start from a state in which the equal profitability condition holds (i.e. $\lambda_{I} V_{I}=\lambda_{C} V_{C}$ ). Suppose the employment in innovative $R \& D$ increases, then, as shown in Table 1 , the increase in the employment in innovative $R \& D$ has two effects: the "business-stealing" effect and the positive competition effect. The "business-stealing" effect tends to lower the values of both innovation and imitation; the positive competition effect does not affect the value of innovation, but it tends to increase the value of imitation. The net result is that the value of innovation decreases more than the value of imitation does. The latter may rise rather than fall if the effective employment in innovative $R \& D$ is low relative to the effective employment in imitative $R \& D$ ! As a result, the expected return to imitative $R \& D$ is higher than that to innovative $\mathrm{R} \& \mathrm{D}$ (i.e. $\lambda_{I} V_{I}<\lambda_{C} V_{C}$ ). The higher expected return to imitative $\mathrm{R} \& \mathrm{D}$ creates an incentive for firms to employ more labor. Then the employment in imitative R\&D rises and the equality of the expected returns to both type of research is restored. Therefore we have the positive relationship between these two types of employment.

The second equation (27) is a labour market clearing condition. The relationship between these two types of employment, $n_{I}$ and $n_{C}$, are negative as shown in Appendix 8. The reason for this negative relationship is obvious. Since the total labour supply $N$ is fixed, then given the employment in the consumption good production, more labour hired by one type of $R \& D$ implies less labour available for the other. We show that as long as the total labour endowment $N$ is large enough and $0<\lambda<\frac{1}{2}$, there always exists an equilibrium with both innovation and imitation. That is, 
Proposition 4: An equilibrium with both innovations and imitations exists if and only if $N>\frac{\rho}{\lambda_{r}(\gamma-1)}$ and $0<\lambda<\frac{1}{2}$. Moreover, the equilibrium is unique if it exists. ${ }^{16}$

The equilibrium properties will be discussed in the next section.

Now we calculate the utility growth rate $g$. We consider the productive imitation case. ${ }^{17}$ Substituting $\Delta_{q_{i}}$ with $p=\gamma \Omega(M) / \Omega\left(M_{-1}\right)$ and $E_{i}=E$ into (12) gives

$$
u(t)=\ln \left(\frac{E}{\gamma}\right)+\int_{0}^{1} q_{i} \ln \gamma d i+\int_{0}^{1} \ln \Omega\left(M_{-1}\right) d i,
$$

where $M_{-1}$ is the number of varieties of vintage $\left(q_{i}-1\right)$ in sector $i$. Using the properties of the Poisson distribution, as in Segerstrom (1991), we have

$$
\begin{aligned}
\int_{0}^{1} q_{i} \ln \gamma d i & =\sum_{q_{i}=0}^{\infty} \frac{\left(\lambda_{I} n_{I} t\right)^{q_{i}} e^{-\lambda_{I} n_{I} t}}{q_{i} !} q_{i} \ln \gamma \\
& =\left(\lambda_{I} n_{I}\right) t \ln \gamma .
\end{aligned}
$$

And the stationary distribution of the type of sector across sectors implies

$$
u_{\nu} \equiv \int_{0}^{1} \ln \Omega\left(M_{-1}\right) d i=\sum_{k_{-1}=1}^{\infty} \theta_{k_{-1}} \ln \Omega\left(k_{-1}\right),
$$

where $k_{-1}$ refers to the type of a sector in the previous generation. Hence, we have

$$
u(t)=\ln \left(\frac{E}{\gamma}\right)+\lambda_{I} n_{I} t \ln \gamma+u_{\nu} .
$$

Then taking the derivative of $\mathrm{u}(\mathrm{t})$ with respect to time $t$ gives the growth rate

$$
g=\left(\lambda_{I} n_{I}\right) \ln \gamma
$$

From (32), we know that the growth rate depends positively on the productivity $\lambda_{I}$ of innovative $R \& D$, the amount of labour $n_{I}$ employed in innovative $R \& D$ and the size $\gamma$ of innovation. Given the parameters, $\lambda_{I}$ and $\gamma$, the growth rate depends solely on the equilibrium

\footnotetext{
${ }^{16}$ See Appendix 8 for proof.

${ }^{17}$ The rent-seeking imitation case can be considered as a special case.
} 
employment $n_{I}$ in innovative $R \& D$. Since the growth rate and the employment in innovative $\mathrm{R} \& \mathrm{D}$ change in the same direction, in the next section, the comparative-statics analysis focuses on the effects of exogenous changes in the model's parameters on the equilibrium employment in innovative $R \& D$ (and the equilibrium employment in imitative $R \& D$ as well).

\section{Comparative Statics And Public Policies}

This section focuses on the equilibrium with both innovation and imitation. We look at the effects of changes in the model's parameters and government policies on the equilibrium employment in innovative and imitative $R \& D$. The effects on welfare will be analyzed through numerical examples in section 6. The effects of exogenous changes in the model's parameters $\left(\lambda_{I}, \lambda_{C}, \rho, \gamma\right.$ and $\left.N\right)$, and of government policies on the employment in innovative and imitative $R \& D$ are summarized in the following Propositions. ${ }^{18}$

Proposition 5: An increase in the productivity $\lambda_{I}$ of innovative $R \& D$ increases the equilibrium employment $n_{I}$ in innovative $\mathrm{R} \& \mathrm{D}$.

The effect of an increase in the productivity of innovative $R \& D$ on the equilibrium employment $n_{I}$ in innovative $R \& D$ is intuitive. The increase in this parameter decreases the marginal cost of innovative R\&D by improving the efficiency of employment. It also decreases the marginal benefit to innovative $R \& D$ because it increases the rate of creative destruction of the next innovation (reinforcing the "business-stealing" effect). But here the former dominates the latter. Consequently, the equilibrium employment in innovative $R \& D$ increases.

\footnotetext{
${ }^{18}$ The proofs are given in Appendix 9, 10 and 11.
} 
Numerical calculations (e.g. Table 4 in Appendix 9) show that an increase in the productivity $\lambda_{I}$ of innovative $R \& D$ decreases (increases) the equilibrium employment $n_{C}$ in imitative $R \& D$ if $\theta_{1} \geq \theta_{1}^{\prime}\left(\theta_{1}<\theta_{1}^{\prime}\right){ }^{19}$ The effect on the equilibrium employment in imitative $R \& D$ needs some explanations. The intuition may suggest that the increase in the productivity of innovative $R \& D$ should always reduce the equilibrium employment in imitative $R \& D$ because it reduces the marginal benefit to imitative $R \& D$ by increasing the rate of creative destruction. But, as has been explained above, the value of imitation depends not only on the length of time in which the imitator enjoys the profit from being the sole producer of a new variety, but also on the distribution of the type of sector across sectors. The increase in the productivity of innovative $R \& D$ reduces the length of time for the imitator to earn profits, but it also increase the probability of copying the state-of-the-art products from those sectors with single producers (the innovators), and therefore has a larger share of that product market (strengthening the positive competition effect). So when the effective employment $\lambda_{I} n_{I}$ in innovative $R \& D$ is low relative to the effective employment $\lambda_{C} n_{C}$ in imitative R\&D, the "business-stealing" effect is more than offset by the positive competition effect. Therefore, the employment in imitative $R \& D$ increases rather than decreases. However, when the effective employment $\lambda_{I} n_{I}$ in innovative $\mathrm{R} \& \mathrm{D}$ is high relative to the effective employment $\lambda_{C} n_{C}$ in imitative $R \& D$, the "business-stealing" effect dominates. Thus, the net effect follows the intuition, that is, the equilibrium employment in imitative $R \& D$ decreases as a result of the productivity increase in innovative R\&D.

Quite symmetrically, for changes in the productivity of imitative $R \& D$, we have

Proposition 6: An increase in the productivity $\lambda_{C}$ of imitative $R \& D$ decreases the equilibrium employment $n_{I}$ in innovative $R \& D$.

\footnotetext{
${ }^{19}$ Here, $\theta_{1}^{\prime}$ is a critical value of $\theta_{1}$.
} 
An increase in the productivity of imitative R\&D does not affect the marginal cost of innovative $R \& D$ but it decreases the marginal benefit to this activity by reducing the length of time during which the innovator enjoys its monopoly profit (increasing the negative competition effect), so the equilibrium employment in innovative $R \& D$ decreases.

It is shown through numerical calculations (e.g. Table 5 in Appendix 9) that an increase in the productivity $\lambda_{C}$ of imitative $R \& D$ increases (decreases) the equilibrium employment $n_{C}$ in imitative R\&D if $\theta_{1}>\theta_{1}^{\prime \prime}\left(\theta_{1} \leq \theta_{1}^{\prime \prime}\right) .{ }^{20}$ The effect on the equilibrium employment in imitative $R \& D$ again depends on two offsetting forces. On the one hand, the increase in the productivity of imitative $R \& D$ reduces the marginal cost of imitative $R \& D$ by improving the efficiency of employment, but on the other hand, it also decreases the marginal benefit to this activity through the negative competition effect. If the effective employment $\lambda_{I} n_{I}$ in innovative $R \& D$ is low relative to the effective employment $\lambda_{C} n_{C}$ in imitative $R \& D$, the negative competition effect dominates and thus, in equilibrium, the employment in imitative $R \& D$ decreases. If the effective employment in innovative $R \& D$ is relatively high, then the effect of the marginal cost decrease more than offsets the negative competition effect. Therefore, the equilibrium employment in imitative R\&D increases.

Proposition 7: An increase in the representative individual's subjective discount rate $\rho$ always decreases the equilibrium employment $n_{C}$ in imitative $R \& D$.

But we show through numerical examples (e.g. Table 6 in Appendix 9) that an increase in the representative individual's subjective discount rate $\rho$ decreases (increases) the equilibrium employment $n_{I}$ in innovative $\mathrm{R} \& \mathrm{D}$ if $\rho$ is large (small). The representative individual's

\footnotetext{
${ }^{20}$ Like $\theta_{1}^{\prime}, \theta_{1}^{\prime \prime}$ is another critical value of $\theta_{1}$.
} 
subjective discount rate is negatively related to the discounted expected benefits to both innovative and imitative $R \& D$. That is, an increase in the discount rate reduces the discounted expected benefit to each type of research. Since it does not affect the marginal cost of either type, the equilibrium employment in innovative and imitative $R \& D$ should decrease responding to lower marginal benefits. This is true if the discount rate is large. However, if the discount rate is small, the increase in the discount rate reduces the equilibrium employment in imitative R\&D to such an extent that the negative competition effect outweighs the discount rate effect on the marginal benefit to innovative $R \& D$. As a result, the employment in innovative $R \& D$ rises rather than falls.

Proposition 8: An increase in the size $\gamma$ of innovation and the labor endowment $N$ increases the equilibrium employment $n_{I}$ in innovative $\mathrm{R} \& \mathrm{D}$ and the equilibrium employment $n_{C}$ in imitative R\&D.

This proposition is very intuitive. An increase in the size of innovation or in the labour endowment raises the marginal benefits to both innovative and imitative $R \& D$. The increase in the the size of innovation increases the marginal benefit through charging a higher price, while the increase in the labour endowment increases the marginal benefit by increasing the total expenditure.

Proposition 9: An increase in the subsidy $s_{I}$ to innovative R\&D increases the equilibrium employment $n_{I}$ in innovative R\&D.

The effect of an increase in the subsidy to innovative $R \& D$ works through the same mechanism discussed in proposition 5. It always induces more employment in innovative R\&D by reducing the marginal cost of innovative R\&D. 
Numerical calculations (e.g. Table 7 in Appendix 9) reveal that an increase in the subsidy $s_{I}$ to innovative $R \& D$ decreases (increases) the equilibrium employment $n_{C}$ in imitative $\mathrm{R} \& \mathrm{D}$ if $\theta_{1} \geq \theta_{1}^{\prime \prime \prime}\left(\theta_{1}<\theta_{1}^{\prime \prime \prime}\right)$. ${ }^{21}$ The effect on the equilibrium employment in imitative $\mathrm{R} \& \mathrm{D}$ again depends on the two conflicting effects mentioned above. If the effective employment in innovative $R \& D$ is low relative to the effective employment in imitative $R \& D$, the positive competition effect is stronger than the "business-stealing" effect, thus the equilibrium employment in imitative $R \& D$ rises; if the effective employment in innovative $R \& D$ is relatively high, the "business-stealing" effect dominates the positive competition effect, therefore the equilibrium employment in imitative $R \& D$ decreases.

Proposition 10: An increase in the subsidy $s_{c}$ to imitative R\&D decreases the equilibrium employment $n_{I}$ in innovative $\mathrm{R} \& \mathrm{D}$ and increases the equilibrium employment $n_{C}$ in imitative R\&D.

This is again an intuitive proposition. An increase in the subsidy to imitative $R \& D$ does not affect the marginal benefit to imitative $R \& D$, but it decreases the marginal cost, thus it induces more employment in imitative $R \& D$. But the increase in the equilibrium employment in imitative $R \& D$ reduces the marginal benefit to innovative $R \& D$ through the negative competition effect. Therefore, responding to the decrease in the marginal benefit, the equilibrium employment in innovative R\&D declines.

Under most circumstances, taxation and subsidization are two alternatives for public policies. That is, taxing an activity can usually be replaced by subsidizing another activity (or other activities) to achieve the same policy objective. However, this does not apply here.

\footnotetext{
${ }^{21} \theta_{1}^{\prime \prime \prime}$ is another critical value of $\theta_{1}$.
} 
In this model, taxation and subsidization are not always equivalent. If the policy objective is to discourage imitations, then taxing imitative $R \& D$ can always satisfy this objective. But subsidizing innovative $R \& D$ may do the opposite. So we have the following corollary.

Corollary: Subsidizing innovations and taxing imitations are not necessarily equivalent.

The results regarding the effects of government subsidies will be compared with those in Segerstrom (1991) in the next section.

\section{Productive Imitation}

In this section, we consider the case where individuals prefer more varieties, i.e. $\Omega^{\prime}(M)>0$. We restrict our attention to the effects on the levels of innovative and imitative R\&D of exogenous changes in the model's parameters and of public policies. To this end, first, we need to calculate the values of innovation and imitation under the new assumption. The comparative statics analysis can be done in the same fashion as in the rent-seeking imitation case.

To derive the value functions of innovation and imitation under the assumption that $\Omega^{\prime}(M)>0$, we need to know the prices innovators and imitators can charge for a new variety or a new product. From the product replacement condition (10), we know that the highest price an innovator or imitator can charge is

$$
p=\beta \Omega(M)
$$

where the subscripts for sectors and varieties are omitted, $\beta \equiv \gamma / \Omega\left(M_{-1}\right)$ and $M$ refers to the number of varieties of the-state-of-the-art product while $M_{-1}$ is the number of varieties of the old product (one step down the quality ladder). Notice that with the assumption that 
$\Omega^{\prime}(M)>0$, the price of a new product (or a variety of a new product) depends not only on the size $\gamma$ of the quality improvement but also on the numbers ( $M$ and $M_{-1}$ ) of varieties of both the new and old products. As in the case where imitations are of the rent-seeking type, the price of a new product increases with the size of the quality improvement. In addition, the price also increases with the number of varieties of the new product but decreases with the number of varieties of the old one. That is, the larger the number of the old (new) varieties, the lower (higher) the price an innovator or imitator can charge. As a result, the values of both innovation and imitation depend crucially on the distribution of the type of sector across sectors. In this case, the calculation of the values of innovation and imitation turns out to be rather complicated. To illustrate the interactions between innovation and imitation in a manageable way, we assume that the function $\Omega(M)$ takes the following simple form

$$
\Omega(M)= \begin{cases}1, & M=1 \\ \nu, & M \geq 2\end{cases}
$$

where $1 \leq \nu \leq \gamma$ represents the individual's preference for varieties. ${ }^{22}$ As has been shown in Appendix 12, the value function of innovation is given by

$$
V_{I}=\left\{\left[\frac{1}{\lambda_{C} n_{C}} \ln \left(1+\frac{\lambda_{C} n_{C}}{\rho+\lambda_{I} n_{I}}\right)\right]\left[\gamma \nu-\theta_{1}-\left(1-\theta_{1}\right) \nu\right]-\frac{(\nu-1)\left[\theta_{1}+\left(1-\theta_{1}\right) \nu\right]}{\rho+\lambda_{I} n_{I}+\lambda_{C} n_{C}}\right\} \frac{E}{\gamma \nu},
$$

and the value function of imitation is given by

$$
\begin{aligned}
V_{C}= & \frac{\lambda_{I} n_{I}}{\rho \lambda_{C} n_{C}}\left[\left(1+\frac{\lambda_{I} n_{I}}{\lambda_{C} n_{C}}\right) \ln \left(1+\frac{\lambda_{C} n_{C}}{\lambda_{I} n_{I}}\right)\right. \\
& \left.-\left(1+\frac{\rho+\lambda_{I} n_{I}}{\lambda_{C} n_{C}}\right) \ln \left(1+\frac{\lambda_{C} n_{C}}{\rho+\lambda_{I} n_{I}}\right)\right]\left[\gamma \nu-\theta_{1}-\left(1-\theta_{1}\right) \nu\right] \frac{E}{\gamma \nu}
\end{aligned}
$$

where $\theta_{1}=\frac{\lambda_{I} n_{I}}{\lambda_{I_{I} n_{I}+\lambda_{C} n_{C}}}$ is the proportion of type 1 sectors.

\footnotetext{
${ }^{22}$ Note that if $\nu=1$, then we have the rent-seeking imitation case.
} 
Comparing (35) and (36) with (19) and (21) respectively, we can see that the assumption that $\Omega^{\prime}(M)>0$ creates two more channels through which the employment in innovative R\&D and imitative $R \& D$ affects the values of innovation and imitation. In addition to the "business-stealing" effect on the value of innovation $V_{I}$ and the "business-stealing" effect and the positive competition effect on the value of imitation $V_{C}$, identified in section 2 , an increase in the employment $n_{I}$ in innovative $R \& D$, on the one hand, increases the profit flows for innovators and imitators by raising the prices they can charge (the positive dynamic competition effect ${ }^{23}$ ) because of (stochastically) fewer old varieties. But on the other hand, the increase in the employment $n_{I}$ in innovative $R \& D$ also affects adversely the profit flows for innovators and imitators by lowering the prices they can charge due to fewer new varieties (the negative dynamic competition effect). Correspondingly, besides the negative competition effect on the values of innovation and imitation, an increase in the employment $n_{C}$ in imitative $R \& D$ has another two conflicting effects on the values of innovation and imitation. It decreases (increases) the values of innovation and imitation by increasing (reducing) the competition between the producers of the products of two different generations (qualities) because of more old varieties (more new varieties)(the negative (positive) dynamic competition effect) which tends to lower (raise) the prices the producers can charge. For comparisons with Table 1 in section 2, we list all these effects in Table 3.

To close the modified model, we consider the labor market. Since each unit of output of any variety and quality costs one unit of labor to produce, the total employment in consumption good production is simply the total output. Let $x$ denote the output of each

\footnotetext{
${ }^{23}$ We distinguish this effect from the positive competition effect because these two effects work through different channels: the positive dynamic competition effect comes from reducing the competition between the producers of a new product and the producers of the new product's old counterpart (old varieties) while the positive competition effect results from reducing the competition among the producers of different varieties of the same generation (quality). For the same reason, we distinguish the negative dynamic competition effect from the negative competition effect.
} 
variety, $M x$ is the employment in a single sector. Therefore, the total employment $X$ is ${ }^{24}$

$$
\begin{aligned}
X & =\int_{0}^{1} x d i=\sum_{k-1}^{\infty}\left[\sum_{k=1}^{\infty}\left(\frac{E}{p}\right) \theta_{k}\right] \theta_{k_{-1}} \\
& =\left[\theta_{1} \nu+\left(1-\theta_{1}\right)\right]\left[\theta_{1}+\left(1-\theta_{1}\right) \nu\right] \frac{E}{\gamma \nu},
\end{aligned}
$$

where $\theta_{k}$ is defined in Appendix 1 and $\theta_{k_{-1}}$ is the measure of sectors that are type $k$ in the previous generation. Thus, we have the labor market clearing condition

$$
n_{I}+n_{C}+\left[\theta_{1} \nu+\left(1-\theta_{1}\right)\right]\left[\theta_{1}+\left(1-\theta_{1}\right) \nu\right] \frac{E}{\gamma \nu}=N .
$$

Therefore, the equilibrium conditions are

$$
\begin{aligned}
& \left\{\left[\frac{\lambda_{I}}{\lambda_{C} n_{C}} \ln \left(1+\frac{\lambda_{C} n_{C}}{\rho+\lambda_{I} n_{I}}\right)\right]\left[\gamma \nu-\theta_{1}-\left(1-\theta_{1}\right) \nu\right]\right. \\
& \left.-\frac{\lambda_{I}(\nu-1)\left[\theta_{1}+\left(1-\theta_{1}\right) \nu\right]}{\rho+\lambda_{I} n_{I}+\lambda_{C} n_{C}}\right\} \frac{E}{\gamma \nu} \leq 1, n_{I} \geq 0, \text { with at least one equality, } \\
& \frac{\lambda_{I} n_{I}}{\rho n_{C}}\left[\left(1+\frac{\lambda_{I} n_{I}}{\lambda_{C} n_{C}}\right) \ln \left(1+\frac{\lambda_{C} n_{C}}{\lambda_{I} n_{I}}\right)-\left(1+\frac{\rho+\lambda_{I} n_{I}}{\lambda_{C} n_{C}}\right) \ln \left(1+\frac{\lambda_{C} n_{C}}{\rho+\lambda_{I} n_{I}}\right)\right] \\
& \\
& {\left[\gamma \nu-\theta_{1}-\left(1-\theta_{1}\right) \nu\right] \frac{E}{\gamma \nu} \leq 1, n_{C} \geq 0, \text { with at least one equality, }}
\end{aligned}
$$

and the above labor market clearing condition (38). In the rest of this section, we will consider equilibria with $n_{I}>0$ and $n_{C}>0$, which are described by

$$
\begin{aligned}
& {\left[\frac{1}{\lambda_{C} n_{C}} \ln \left(1+\frac{\lambda_{C} n_{C}}{\rho+\lambda_{I} n_{I}}\right)\right]\left[\gamma \nu-\theta_{1}-\left(1-\theta_{1}\right) \nu\right]-\frac{(\nu-1)\left[\theta_{1}+\left(1-\theta_{1}\right) \nu\right]}{\rho+\lambda_{I} n_{I}+\lambda_{C} n_{C}}} \\
& \quad=\frac{n_{I}}{\rho n_{C}}\left[\left(1+\frac{\lambda_{I} n_{I}}{\lambda_{C} n_{C}}\right) \ln \left(1+\frac{\lambda_{C} n_{C}}{\lambda_{I} n_{I}}\right)-\left(1+\frac{\rho+\lambda_{I} n_{I}}{\lambda_{C} n_{C}}\right) \ln \left(1+\frac{\lambda_{C} n_{C}}{\rho+\lambda_{I} n_{I}}\right)\right] \\
& \quad\left[\gamma \nu-\theta_{1}-\left(1-\theta_{1}\right) \nu\right],
\end{aligned}
$$

and

\footnotetext{
${ }^{24}$ See Appendix 13 for derivation.
} 
Table 3: The Effects of Changes in $n_{I}$ and $n_{C}$ with $\Omega^{\prime}(M)>0$

\begin{tabular}{cll}
\hline \hline Employ.Increase & Effect on $V_{I}$ & Effect on $V_{C}$ \\
\hline$n_{I}$ & "Business-stealing" effect & "Business-stealing" effect \\
& $\Rightarrow V_{I}$ decreases & $\Rightarrow V_{C}$ decreases \\
& Positive dynamic & Positive dynamic \\
competition effect & competition effect \\
$\Rightarrow V_{I}$ increases & $\Rightarrow V_{C}$ increases \\
& Negative dynamic & Negative dynamic \\
competition effect & competition effect \\
& $\Rightarrow V_{I}$ decreases & $\Rightarrow V_{C}$ decreases \\
& & Positive competition effect \\
& $\Rightarrow V_{C}$ increases \\
\hline$n_{C}$ & Negative competition effect & Negative competition effect \\
& $\Rightarrow V_{I}$ decreases & $\Rightarrow V_{C}$ decreases \\
& Positive dynamic & Positive dynamic \\
& competition effect & competition effect \\
& $\Rightarrow V_{I}$ increases & $\Rightarrow V_{C}$ increases \\
& Negative dynamic & Negative dynamic \\
& competition effect & competition effect \\
& $\Rightarrow V_{I}$ decreases & $\Rightarrow V_{C}$ decreases \\
\hline
\end{tabular}

Note: As in Table 1, this table does not list the effects of increases in $n_{I}$ and $n_{C}$ on $V_{I}$ and $V_{C}$ by reducing the employment in production and thus decreasing the profit flow for each producer. 


$$
\begin{aligned}
& \left\{\left[\frac{\lambda_{I}}{\lambda_{C} n_{C}} \ln \left(1+\frac{\lambda_{C} n_{C}}{\rho+\lambda_{I} n_{I}}\right)\right]\left[\gamma \nu-\theta_{1}-\left(1-\theta_{1}\right) \nu\right]\right. \\
& \left.\quad-\frac{(\nu-1)\left[\theta_{1}+\left(1-\theta_{1}\right) \nu\right]}{\rho+\lambda_{I} n_{I}+\lambda_{C} n_{C}}\right\} \frac{\left(N-n_{I}-n_{C}\right)}{\left[\theta_{1} \nu+\left(1-\theta_{1}\right)\right]\left[\theta_{1}+\left(1-\theta_{1}\right) \nu\right]}=1 .
\end{aligned}
$$

Since innovation and imitation interact with each other in such complicated ways, as shown in Table 3, we do not attempt to do comparative-statics and public policy experiments analytically. Instead, numerical examples are used. The numerical examples give us the following results:

For the changes in $\lambda_{I}, \lambda_{C}, \gamma, \rho, N$ and $s_{I},{ }^{25}$ we have qualitatively the same results as stated in section 4 (i.e. the case where imitations are of the rent-seeking type). The effects of changes in $\nu$ and $s_{C}$ on the employment in innovative and imitative $\mathrm{R} \& \mathrm{D}$ are as follows

- An increase in the degree $\nu$ of preference for more varieties decreases the employment $n_{I}$ in innovative $\mathrm{R} \& \mathrm{D}$ and it may increase or decrease the employment $n_{C}$ in imitative R\&D. For example, when $\left(\lambda_{I}, \lambda_{C}, \gamma, \rho, N\right)=(0.3,3,2,0.05,1)$, an increase in the degree $\nu$ of preference for varieties from 1 to 1.1 decreases the employment $n_{I}$ in innovative R\&D from $0.020,882$ to $0.019,221$ and also decreases the employment $n_{C}$ in imitative R\&D from $0.190,179$ to $0.183,109$; when $\left(\lambda_{I}, \lambda_{C}, \gamma, \rho, N\right)=(1.3,3,2,0.05,1)$, if the degree $\nu$ of preference for varieties increases from 1 to 1.1, then the employment $n_{I}$ in innovative $R \& D$ drops from $0.322,868$ to $0.263,759$, but the employment $n_{C}$ in imitative $R \& D$ rises from $0.156,196$ to $0.211,520$.

- An increase in the subsidy $s_{C}$ to imitative R\&D decreases the employment $n_{I}$ in innovative $R \& D$ and it may increase or decrease the employment $n_{C}$ in imitative $R \& D$. For

\footnotetext{
${ }^{25}$ With a subsidy $s_{I}$ to innovative $R \& D$, the equilibrium conditions are given by (41) with its left hand side being multiplied by $\frac{1}{1-s_{I}}$ and (42) with its right hand side being replaced by $1-s_{I}$; the equilibrium with a subsidy $s_{C}$ to imitative R\&D is described by (42) and (41) with its right hand side being multiplied by $\frac{1}{1-s c}$.
} 
example, when $\left(\lambda_{I}, \lambda_{C}, \gamma, \rho, \nu, N\right)=(0.1,0.3,2,0.05,1.5,1)$, an increase in the subsidy $s_{C}$ to imitative $\mathrm{R} \& \mathrm{D}$ from zero to 0.01 decreases the employment $n_{I}$ in innovative $\mathrm{R} \& \mathrm{D}$ from $0.011,770$ to $0.011,422$ and also decreases the employment $n_{C}$ in imitative $R \& D$ from $0.032,141$ to $0.031,891$. When $\left(\lambda_{I}, \lambda_{C}, \gamma, \rho, \nu, N\right)=(0.3,3,2,0.05,1.5,1)$, without a subsidy $s_{C}$ to imitative $R \& D$, the employment $n_{I}\left(n_{C}\right)$ in innovative (imitative) $\mathrm{R} \& \mathrm{D}$ is $0.267,863(0.387,805)$; with a subsidy $s_{C}=0.01$, the employment $n_{I}\left(n_{C}\right)$ in innovative (imitative) $R \& D$ decreases (increases) to $0.262,566(0.393,147)$.

The above numerical calculations show that when imitations are productive, the "nonequivalence" result still applies; ${ }^{26}$ in addition, taxing imitative R\&D may induce more imitations! The reason for this seemingly counterintuitive result is as follows. If imitative $R \& D$ is taxed, then the marginal cost of imitative $R \& D$ will rise. Meanwhile, the employment in innovative $R \& D$ will increase. As listed in Table 3 , the increase in the employment in innovative $R \& D$ has both positive and negative effects on the value of imitation. But with certain sets of parameters, the positive effects dominate the negative effects and increases the marginal benefit to imitative $R \& D$. If the increase in the marginal benefit is greater than that in the marginal cost, then the employment in imitative $R \& D$ will rise rather than fall.

Let us compare the results regarding government subsidies with those in Segerstrom (1991). ${ }^{27}$ Before doing so, it is helpful to calculate the aggregate employment in innovative and imitative R\&D and the average growth rate in Segerstrom's model. Using the notations in the present paper, we know that, in his model, $n_{I}=a_{I}\left(\frac{C}{I+C}\right) I, n_{C}=a_{C}\left(\frac{I}{I+C}\right) C$ and $g=\frac{1}{a_{I}} n_{I} \ln \lambda$, where $a_{I}\left(a_{C}\right)$ is the amount of labor required for each unit of innovative (imitative) R\&D activity, $I(C)$ is the level of innovative (imitative) $\mathrm{R} \& \mathrm{D}$ in a single industry

\footnotetext{
${ }^{26}$ The "nonequivalence" result refers to the corollary in section 4.

${ }^{27}$ As has been introduced at the beginning of the present paper, in Segerstrom's model, a steady state equilibrium is defined in a way such that some industries are targeted by innovative $R \& D$ and the others are targeted by imitative $R \& D$, so innovations and imitations can not occur in the same industry at the same time.
} 
(sector) if this industry is targeted by innovative (imitative) $R \& D$, and $\lambda$ is equivalent to $\gamma$ in the present paper. We can easily see that, in Segerstrom's model, the aggregate employment in innovative $R \& D$ and that in imitative $R \& D$ always change in the same direction. Furthermore, the former is always proportionally greater that the latter because of the assumption that $a_{I}>a_{C}$. This property comes directly from the definition of equilibrium. In present model, the employment levels of innovative and imitative $R \& D$ are determined by the costs and benefits of these two activities, therefore, they may or may not change in the same direction.

Now let us see the differences in the effects of government subsidies on the employment in innovative and imitative R\&D. While both Segerstrom's and the present model (in both the rent-seeking and productive imitation cases) show that an increase in the subsidy $s_{I}$ to innovative $R \& D$ always increases the employment in innovative $R \& D$ and thus the growth rate, these two models have different results concerning the effect on the employment in imitative R\&D. Since, in Segerstrom's model, the employment in imitative R\&D changes in the same direction as the employment in innovative $R \& D$ does, the employment in imitative increases with the increase in the subsidy $s_{I}$. But the present model shows that in both the rent-seeking and productive imitation cases, responding to an increase in the subsidy $s_{I}$, the employment in imitative R\&D may increase or decrease.

As to the effects of an increase in the subsidy $s_{C}$ to imitative R\&D on the employment in innovative and imitative $R \& D$ and the growth rate, the two models also have different results. Segerstrom shows that the effects of an increase in the subsidy to imitative R\&D ambiguously affects the employment in innovative and imitative $R \& D$ and thus the growth rate; we show that an increase in the subsidy $s_{C}$ always decreases the employment in innovative $R \& D$ and consequently the growth rate in either the rent-seeking imitation or the 
productive imitation case, and unambiguously increases the employment in imitative $R \& D$ when imitations are of the rent-seeking type, although, as in Segerstrom's model, the effect on the employment in imitative $R \& D$ can go either way when imitations are productive.

\section{Welfare}

In this section, we analyze the effects of changes in government policies on the consumer's welfare. Before doing so, we need to calculate the consumer's discounted expected life time utility. Since the rent-seeking imitation case can be considered as a limiting case of the productive imitation case, we just need to consider the productive imitation case. From equation (30) in section 3 and the assumption in section 5 that $\Omega(M)=1$ if $M=1$ and $\Omega(M)=\nu$ if $M \geq 2$, we have

$$
u_{\nu}=\left(1-\theta_{1}\right) \ln \nu
$$

Then substituting (43) and (31) into (13) gives

$$
U=\frac{1}{\rho}\left[\ln \left(\frac{E}{\gamma}\right)+u_{\nu}+\frac{\lambda_{I} n_{I} \ln \gamma}{\rho}\right]
$$

where $\theta_{1}$ is defined in Appendix 1 and the total expenditure, $E$, must satisfy the labor market clearing condition (36), or equivalently,

$$
\frac{E}{\gamma}=\frac{\nu\left(N-n_{I}-n_{C}\right)}{\left[\theta_{1} \nu+\left(1-\theta_{1}\right)\right]\left[\theta_{1}+\left(1-\theta_{1}\right) \nu\right]} \text {. }
$$

Notice that if $\nu=1$ (i.e. imitations are of the rent-seeking type), then (44) becomes

$$
U=\frac{1}{\rho}\left[\ln \left(\frac{E}{\gamma}\right)+\frac{\lambda_{I} n_{I} \ln \gamma}{\rho}\right],
$$

where $E / \gamma=\left(N-n_{I}-n_{C}\right)$. 
It is clear from (32) that imitations do not contribute to growth. But we know from (44) that except in the case where $\Omega(M)=1$ (i.e. $\nu=1$ ), they do improve welfare by providing the consumer with more varieties. Since the welfare depends on current consumption $E$, the number of varieties (indirectly represented by $u_{\nu}$ ) and the growth rate $g$ (i.e. $\lambda_{I} n_{I} \ln \gamma$ ), growth and welfare may change in different directions. That is, a higher growth rate does not necessarily implies a higher level of welfare. Therefore, a government policy leading to a higher growth rate may reduce welfare.

As has been shown in sections 4 and 5 , an increase in the subsidy to innovative (imitative) $R \& D$ increases (decreases) the employment in innovative $R \& D$, so it speeds up (slows down) economic growth. However, numerical examples show that an increase in the subsidy $s_{I}$ to innovative $\mathrm{R} \& \mathrm{D}$ has an ambiguous effect on welfare. When $\left(\lambda_{I}, \lambda_{C}, \gamma, \rho, \nu, N\right)=(0.1$, $0.3,5,0.05,1.5,1)$, an increase in the subsidy $s_{I}$ from zero to 0.01 raises welfare, but when $\left(\lambda_{I}, \lambda_{C}, \gamma, \rho, \nu, N\right)=(0.1,0.3,2,0.05,1.5,1)$, the same policy change lowers welfare

The effect on welfare of an increase in the subsidy $s_{C}$ to imitative $R \& D$ is also ambiguous. For example, when $\left(\lambda_{I}, \lambda_{C}, \gamma, \rho, \nu, N\right)=(0.1,0.21,2,0.05,1.5,1)$, an increase in the subsidy $s_{C}$ from zero to 0.01 increases welfare, but when $\left(\lambda_{I}, \lambda_{C}, \gamma, \rho, \nu, N\right)=(0.1,0.21,5$, $0.05,1.5,1)$, the same policy change does the opposite. These results are quite similar to those obtained by Segerstrom (1991).

\section{Concluding Remarks}

This paper has developed a multi-sector dynamic general equilibrium growth model, in which investments in both innovative and imitative $R \& D$ are endogenously determined and economic growth is driven by innovation through its interactions with imitation. Different from 
the endogenous growth literature, the processes of innovation and imitation are modelled in such a way that innovations and imitations can occur in the same sector at the same time, which we believe is consistent with casual observations.

To understand the relationship between innovation and imitation, we identify the channels through which innovation and imitation interact with each other. We consider both rent-seeking and productive imitations. When imitations are of the rent-seeking type, we show that subsidizing innovative $R \& D$ is not necessarily equivalent to taxing imitative $R \& D$; When imitations are productive, we show through numerical examples that, in addition to the "nonequivalence" result, taxing imitative $R \& D$ may induce more employment in imitative $R \& D$. We also show that, in both cases, a subsidy to innovative $R \& D$ always speeds up economic growth while a subsidy to imitative $R \& D$ always does the opposite, but the effects on welfare of both subsidies are ambiguous.

We believe that the model captures many important aspects of a real world economy, especially the complicated interactions between innovation and imitation. It can be extended to study several other issues. For example, patent enforcement can be introduced into the model along the line of Dividson and Segerstrom (1993) to see how patent enforcement affects economic growth through influencing the investment in innovative and imitative $R \& D$. Another promising area is international trade. By extending the model to the context of an open economy, we can analyze the impact of international trade on the investment in innovative and imitative R\&D and therefore on a country's growth rate and the roles of government public and commercial policies in stimulating the country's economic growth. 


\section{Appendix}

\section{Appendix 1: Stationary Distribution of the Type of Sector}

This appendix shows that the random variable $(K-1)$ follows a geometric distribution. Let $\theta_{k}$ be the proportion of type $k$ sectors, then we have

$$
\begin{aligned}
& \dot{\theta}_{1}=\left(1-\theta_{1}\right) \lambda_{I} n_{I}-\theta_{1} \lambda_{C} n_{C} \\
& \dot{\theta}_{k}=\theta_{k-1} \lambda_{C} n_{C}-\theta_{k}\left[\lambda_{I} n_{I}+\lambda_{C} n_{C}\right], \quad k=2,3, \ldots
\end{aligned}
$$

Stationary distribution $\left(\dot{\theta}^{\prime} s=0\right)$ implies

$$
\begin{aligned}
& \theta_{1}=\frac{\lambda_{I} n_{I}}{\lambda_{I} n_{I}+\lambda_{C} n_{C}}, \\
& \theta_{k}=\left(\frac{\lambda_{C} n_{C}}{\lambda_{I} n_{I}+\lambda_{C} n_{C}}\right)^{k-1} \theta_{1}, \quad k=2,3, \ldots .
\end{aligned}
$$

Obviously, $(K-1)$ has a geometric distribution.

\section{Appendix 2: Derivation of $V_{I}$ and $V_{C}$}

We derive the value functions of innovation and imitation in this appendix. Let $V_{C i}$ be the value of the $i$ th successful imitation, then we have the Bellman equation

$$
\rho V_{C i}=\left(\frac{\gamma-1}{\gamma}\right) E /(1+i)-\lambda_{I} n_{I} V_{C i}-\lambda_{C} n_{C}\left(V_{C i}-V_{C(i+1)}\right),
$$

which implies

$$
V_{C(i+1)}=\frac{\left(\rho+\lambda_{I} n_{I}+\lambda_{C} n_{C}\right) V_{C i}-\left(\frac{\gamma-1}{\gamma}\right) E /(i+1)}{\lambda_{C} n_{C}} .
$$

Let $\phi_{i} \equiv \frac{\left(\frac{\gamma-1}{\gamma}\right) E /(1+i)}{\lambda_{C} n_{C}}$ and $\psi \equiv \frac{\rho+\lambda_{Y} n_{I}+\lambda_{C} n_{C}}{\lambda_{C} n_{C}}>1$, then (47) becomes

$$
V_{C i}=\psi V_{C(i-1)}-\phi_{i-1}
$$




$$
=\psi^{i-1} V_{C 1}-\sum_{j=1}^{i-1} \psi^{i-1-j} \phi_{j}
$$

Or equivalently,

$$
\frac{V_{C i}}{\psi^{i-1}}=V_{C 1}-\sum_{j=1}^{i-1} \frac{\phi_{j}}{\psi^{j}}
$$

Since the value of a new successful imitation decreases as more imitations succeed and approaches zero as the number of successful imitations goes to infinity, we have

$$
\begin{aligned}
0=\lim _{i \rightarrow \infty} & \frac{V_{C i}}{\psi^{i-1}}=V_{C 1}-\lim _{i \rightarrow \infty} \sum_{j=1}^{i-1} \frac{\phi_{j}}{\psi^{j}} \\
& =V_{C 1}-\frac{\left(\frac{\gamma-1}{\gamma}\right) E}{\lambda_{C} n_{C}}\left[\psi \ln \left(\frac{\psi}{\psi-1}\right)-1\right],
\end{aligned}
$$

which gives

$$
V_{C 1}=\left[\psi \ln \left(\frac{\psi}{\psi-1}\right)-1\right] \frac{\left(\frac{\gamma-1}{\gamma}\right) E}{\lambda_{C} n_{C}} .
$$

From the Bellman equation for the value of innovation, i.e.

$$
\rho V_{I}=\left(\frac{\gamma-1}{\gamma}\right) E-\lambda_{I} n_{I} V_{I}-\lambda_{C} n_{C}\left(V_{I}-V_{C 1}\right)
$$

we have

$$
V_{I}=\frac{\left(\frac{\gamma-1}{\gamma}\right) E+\lambda_{C} n_{C} V_{C 1}}{\rho+\lambda_{I} n_{I}+\lambda_{C} n_{C}}
$$

Then substituting $V_{C 1}$ with $\psi=\frac{\rho+\lambda_{r} n_{C}+\lambda_{C} n_{C}}{\lambda_{C} n_{C}}$ into (50), we get the value function of innovation

$$
V_{I}=\left[\frac{1}{\lambda_{C} n_{C}} \ln \left(1+\frac{\lambda_{C} n_{C}}{\rho+\lambda_{I} n_{I}}\right)\right]\left(\frac{\gamma-1}{\gamma}\right) E .
$$

Now we derive the value function of imitation. From (48) and (49), we have

$$
V_{C i}=\alpha_{i} \frac{\left(\frac{\gamma-1}{\gamma}\right) E}{\lambda_{C} n_{C}}
$$


where $\alpha_{i}=\psi^{i-1}\left[\psi \ln \left(\frac{\psi}{\psi-1}\right)-1\right]-\sum_{j=1}^{i-1} \psi^{i-1-j} \frac{1}{1+j}$. Let $\delta \equiv \frac{\lambda_{a} n_{c}}{\lambda_{I_{I}}+\lambda_{C} n_{C}}$, then the assumption that imitations occur randomly and independently across sectors implies

$$
\begin{aligned}
V_{C} & =\sum_{i=1}^{\infty} \theta_{i} V_{C i} \\
& =\left(\sum_{i=1}^{\infty} \delta^{i-1} \alpha_{i}\right) \frac{\left(\frac{\gamma-1}{\gamma}\right) E \theta_{1}}{\lambda_{C} n_{C}}
\end{aligned}
$$

Using $\ln \left(1-\frac{1}{\psi}\right)=-\sum_{i=1}^{\infty} \frac{(1 / \psi)^{i}}{i}$, and $\psi \delta>1$, we get

$$
\begin{aligned}
& \sum_{i=1}^{\infty} \delta^{i-1} \alpha_{i}=\psi\left(\frac{(1 / \psi)^{2}}{2}+\frac{(1 / \psi)^{3}}{3}+\frac{(1 / \psi)^{4}}{4}+\ldots\right) \\
& +\psi^{2} \delta\left(\frac{(1 / \psi)^{3}}{3}+\frac{(1 / \psi)^{4}}{4}+\ldots\right) \\
& +\psi^{3} \delta^{2}\left(\frac{(1 / \psi)^{4}}{4}+\frac{(1 / \psi)^{5}}{5}+\ldots\right)+\ldots \\
& =\frac{1}{2 \psi}+\frac{\delta}{3 \psi}+\frac{\delta^{2}}{4 \psi}+\ldots \\
& +\frac{1}{3 \psi^{2}}+\frac{\delta}{4 \psi^{2}}+\frac{\delta^{2}}{5 \psi^{2}}+\ldots \\
& +\frac{1}{4 \psi^{3}}+\frac{\delta}{5 \psi^{3}}+\frac{\delta^{2}}{6 \psi^{3}}+\ldots \\
& +\ldots \\
& =\frac{1}{\psi \delta^{2}}[-\ln (1-\delta)-\delta] \\
& +\frac{1}{\psi^{2} \delta^{3}}\left[-\ln (1-\delta)-\delta-\frac{\delta^{2}}{2}\right] \\
& +\frac{1}{\psi^{3} \delta^{4}}\left[-\ln (1-\delta)-\delta-\frac{\delta^{2}}{2}-\frac{\delta^{3}}{3}\right]+\ldots \\
& =-\left[\frac{\ln (1-\delta)}{\delta} \frac{1}{\psi \delta-1}+\frac{1}{\psi \delta-1}+\frac{1}{2 \psi} \frac{1}{\psi \delta-1}+\frac{1}{3 \psi^{2}} \frac{1}{\psi \delta-1}+\ldots\right] \\
& =\frac{1}{\psi \delta-1}\left[\frac{1}{\delta} \ln \left(\frac{1}{1-\delta}\right)+\psi \ln \left(1-\frac{1}{\psi}\right)\right] \text {. }
\end{aligned}
$$

Substituting $\psi, \delta$ and (53) into (52) gives 


$$
\begin{aligned}
V_{C}= & \frac{\lambda_{I} n_{I}}{\rho \lambda_{C} n_{C}}\left[\left(1+\frac{\lambda_{I} n_{I}}{\lambda_{C} n_{C}}\right) \ln \left(1+\frac{\lambda_{C} n_{C}}{\lambda_{I} n_{I}}\right)\right. \\
& \left.-\left(1+\frac{\rho+\lambda_{I} n_{I}}{\lambda_{C} n_{C}}\right) \ln \left(1+\frac{\lambda_{C} n_{C}}{\rho+\lambda_{I} n_{I}}\right)\right]\left(\frac{\gamma-1}{\gamma}\right) E .
\end{aligned}
$$

This is the value function of imitation shown in section 2 .

\section{Appendix 3: Effects of $n_{I}$ and $n_{C}$ on $V_{I}$}

From the value function of innovation (19) (given $E$ ), we have

$$
\begin{aligned}
& \frac{\partial V_{I}}{\partial n_{I}}=-\frac{\lambda_{I}\left(\frac{\gamma-1}{\gamma}\right) E}{\left(\rho+\lambda_{I} n_{I}\right)\left(\rho+\lambda_{I} n_{I}+\lambda_{C} n_{C}\right)}<0, \\
& \frac{\partial V_{I}}{\partial n_{C}}=-\frac{1}{\lambda_{C} n_{C}^{2}}\left[\ln \left(1+\frac{\lambda_{C} n_{C}}{\rho+\lambda_{I} n_{I}}\right)-\frac{\lambda_{C} n_{C}}{\rho+\lambda_{I} n_{I}+\lambda_{C} n_{C}}\right]\left(\frac{\gamma-1}{\gamma}\right) E<0 .
\end{aligned}
$$

The second inequality is true because

$$
\ln \left(1+\frac{\lambda_{C} n_{C}}{\rho+\lambda_{I} n_{I}}\right)>\frac{\lambda_{C} n_{C}}{\rho+\lambda_{I} n_{I}+\lambda_{C} n_{C}} .
$$

\section{Appendix 4: Effects of $n_{I}$ and $n_{C}$ on $V_{C}$}

For simplicity, we use the following notations: $x=\lambda_{I} n_{I} / \rho, s=\left(\lambda_{I} n_{I}+\lambda_{C} n_{C}\right) / \rho$, and $\lambda=\lambda_{I} / \lambda_{C}$. Then the value function of imitation (21) can be rewritten as

$$
V_{C}=\frac{x}{\rho(s-x)^{2}}\left[s \ln \left(\frac{s}{x}\right)-(s+1) \ln \left(\frac{s+1}{x+1}\right)\right]\left(\frac{\gamma-1}{\gamma}\right) E .
$$

Then given $E$, we get

$$
\frac{\partial V_{C}}{\partial n_{I}}=\frac{\lambda_{I}}{\rho^{2}(s-x)^{2}}\left[(s+x) \ln \left(\frac{s}{x}\right)-(x+s+1) \ln \left(\frac{s+1}{x+1}\right)-\frac{s-x}{x+1}\right]\left(\frac{\gamma-1}{\gamma}\right) E .
$$

We claim that there exists $0<x^{\circ}<s$ (or equivalently $n_{I}^{0}>0$ ) such that 


$$
\frac{\partial V_{I}}{\partial n_{I}}\left\{\begin{array}{l}
>0, \quad 0<x<x^{\circ} \\
=0, \quad x=x^{\circ} \\
<0, \quad s>x>x^{\circ}
\end{array}\right.
$$

Note that given the model's parameters, a higher value of $x^{\circ}$ is uniquely associated with a higher level of effective employment in innovative R\&D.

\section{Proof: Define}

$$
f(x) \equiv(s+x) \ln \frac{s}{x}-(s+x+1) \ln \frac{s+1}{x+1}-\frac{s-x}{x+1} .
$$

Then

$$
f^{\prime}(x)=\ln \frac{s(x+1)}{x(s+1)}-\frac{s-x}{x(x+1)^{2}}
$$

and

$$
f^{\prime \prime}(x)=\frac{1}{x^{2}(x+1)^{3}}\left[(s-x)(3 x+1)-x^{2}(x+1)\right]
$$

Obviously, we have

$$
f^{\prime \prime}(x)\left\{\begin{array}{l}
>0,0<x<x^{\prime \prime} \\
<0, \quad s>x>x^{\prime \prime}
\end{array}\right.
$$

where $0<x^{\prime \prime}<0$ satisfies $f^{\prime \prime}\left(x^{\prime \prime}\right)=0$, i.e. $x^{\prime \prime}\left[1+\frac{x^{\prime \prime}\left(x^{\prime \prime}+1\right)}{3 x^{\prime \prime}+1}\right]=s$. In addition,

$$
\begin{aligned}
\lim _{x \rightarrow 0} f^{\prime}(x) & =\lim _{x \rightarrow 0}\left[\ln \frac{s(x+1)}{x(s+1)}-\frac{s-x}{x(x+1)^{2}}\right] \\
= & \lim _{x \rightarrow 0} \frac{s-x}{x(x+1)^{2}}\left[\frac{\ln \frac{s(x+1)}{x(s+1)}}{\frac{s-x}{x(x+1)^{2}}}-1\right]=-\infty,
\end{aligned}
$$

because

$$
\lim _{x \rightarrow 0} \frac{\ln \frac{s(x+1)}{x(s+1)}}{\frac{s-x}{x(x+1)^{2}}}=\lim _{x \rightarrow 0} \frac{x(x+1)^{2}}{x(x+1)+(s-x)(3 x+1)}=0
$$

and

$$
\lim _{x \rightarrow s} f^{\prime}(x)=\lim _{x \rightarrow s}\left[\ln \frac{s(x+1)}{x(s+1)}-\frac{s-x}{x(x+1)^{2}}\right]=0
$$


Then (57), (58) and (59) implies

$$
f^{\prime}(x)\left\{\begin{array}{l}
<0,0<x<x^{\prime} \\
>0, \quad s>x>x^{\prime}
\end{array}\right.
$$

where $0<x^{\prime}<s$ satisfies $f^{\prime}\left(x^{\prime}\right)=0$, i.e. $\ln \frac{s\left(x^{\prime}+1\right)}{x^{\prime}(s+1)}-\frac{s-x^{\prime}}{x^{\prime}\left(x^{\prime}+1\right)^{2}}=0$. Furthermore,

$$
\lim _{x \rightarrow 0} f(x)=\lim _{x \rightarrow 0}\left[\ln \frac{s}{x}-(s+x+1) \ln \frac{s+1}{x+1}-\frac{s-x}{x+1}\right]=\infty,
$$

and

$$
\lim _{x \rightarrow s} f(x)=\lim _{x \rightarrow s}\left[\ln \frac{s}{x}-(s+x+1) \ln \frac{s+1}{x+1}-\frac{s-x}{x+1}\right]=0 .
$$

Combining (61), (62) with (60), we have

$$
f(x)\left\{\begin{array}{l}
>0, \quad 0<x<x^{\circ} \\
=0, \quad x=x^{\circ} \\
<0, \quad s>x>x^{\circ}
\end{array}\right.
$$

where $0<x^{\circ}<s$. Then (63) is equivalent to (56). Q.E.D.

From (55), we also have

$$
\frac{\partial V_{C}}{\partial n_{C}}=-\frac{\lambda_{C} x}{\rho^{2}(s-x)^{3}}\left[(x+s) \ln \left(\frac{s}{x}\right)-(x+s+2) \ln \left(\frac{s+1}{x+1}\right)\right]\left(\frac{\gamma-1}{\gamma}\right) E<0,
$$

because we can show that

$$
f(s) \equiv(x+s) \ln \left(\frac{s}{x}\right)-(x+s+2) \ln \left(\frac{s+1}{x+1}\right)>0, \forall s>x>0 .
$$

Obviously, $f(s=x)=0$. Moreover, we have

$$
\begin{aligned}
& f^{\prime}(s)=\ln \left[\frac{s(x+1)}{x(s+1)}\right]-\frac{s-x}{s(s+1)}>0, \text { because } \\
& \ln \left[\frac{s(x+1)}{x(s+1)}\right]>\frac{s-x}{s(s+1)} .
\end{aligned}
$$

Thus $f(s=x)=0$ and $f^{\prime}(s)>0$ are sufficient for (64) to hold. 


\section{Appendix 5: Proof of Proposition 1}

Proof: 1. If $N \leq \frac{\rho}{\lambda_{I}(\gamma-1)}$ and/or $\lambda=0$, then $n_{I}=n_{C}=0$.

(i). If $N \leq \frac{\rho}{\lambda_{I}(\gamma-1)}$, then

$$
\begin{aligned}
\max \lambda_{I} V_{I} & =\lim _{s \rightarrow x} \frac{\lambda_{I}}{\rho(s-x)}\left(\ln \frac{s+1}{x+1}\right)(\gamma-1)\left(N-n_{I}-n_{C}\right) \\
& =\frac{\lambda_{I}(\gamma-1)\left(N-n_{I}\right)}{\rho+\lambda_{I} n_{I}} \leq \frac{\rho}{\rho+\lambda_{I} n_{I}}-\frac{\lambda_{I}(\gamma-1) n_{I}}{\rho+\lambda_{I} n_{I}}<1, \text { if } n_{I}>0 .
\end{aligned}
$$

So, $n_{I}=0$. But if $n_{I}=0$, then $V_{C}=0$ because

$$
\lim _{x \rightarrow 0} V_{C}=\lim _{x \rightarrow 0} \frac{x}{\rho(s-x)^{2}}\left[s \ln \left(\frac{s}{x}\right)-(s+1) \ln \left(\frac{s+1}{x+1}\right)\right]\left(\frac{\gamma-1}{\gamma}\right)\left(N-n_{I}-n_{C}\right)=0 .
$$

Equation (65) is true because

$$
\lim _{x \rightarrow 0} x \ln \frac{s}{x}=0 \text { and } \lim _{x \rightarrow 0} x \ln \frac{s+1}{x+1}=0 .
$$

Thus $n_{C}=0$, therefore, $n_{I}=n_{C}=0$.

(ii). If $\lambda=0$, then $\lambda_{I} V_{I}=0<1$, so $n_{I}=0$. From (i), we know that $n_{I}=0$ implies $n_{C}=0$. Therefore, $n_{I}=n_{C}=0$.

2. If $n_{I}=n_{C}=0$, then $N \leq \frac{\rho}{\lambda_{I}(\gamma-1)}$ and/or $\lambda=0$.

If $n_{C}=0$, then $V_{I}=\frac{\lambda_{I}(\gamma-1)\left(N-n_{I}\right)}{\rho+\lambda_{I} n_{I}}$. Then if $n_{I}=0$, then we have

$$
\frac{\lambda_{I}(\gamma-1) N}{\rho} \leq 1, \text { which implies }
$$

$N \leq \frac{\rho}{\lambda_{I}(\gamma-1)}$ and/or $\lambda=0$. Q.E.D. 


\section{Appendix 6: Proof of Proposition 2}

Proof: 1 . If $N>\frac{\rho}{\lambda_{I}(\gamma-1)}$ and $\lambda \geq 1 / 2$, then $n_{I}>0$ and $n_{C}=0$.

(i). Show if $\lambda \geq 1 / 2$, then $\lambda_{I} V_{I}>\lambda_{C} V_{C} \forall s>x>0$. From (19) and (21), we have

$$
\lambda_{I} V_{I}-\lambda_{C} V_{C}=\frac{\lambda_{C} x s}{\rho(s-x)^{2}}\left[\left(1+\frac{\lambda}{x}+\frac{1-\lambda}{s}\right) \ln \frac{s+1}{x+1}-\ln \frac{s}{x}\right](\gamma-1)\left(N-n_{I}-n_{C}\right) .
$$

Define

$$
f(s, x, \lambda) \equiv\left(1+\frac{\lambda}{x}+\frac{1-\lambda}{s}\right) \ln \frac{s+1}{x+1}-\ln \frac{s}{x} .
$$

Since

$$
\frac{\partial f}{\partial \lambda}=\left(\frac{1}{x}-\frac{1}{s}\right) \ln \frac{s+1}{x+1}>0, \forall s>x>0
$$

if $f(s, x, 1 / 2)>0, \forall s>x>0$, then $f(s, x, \lambda)>0, \forall \lambda \geq 1 / 2, s>x>0$. Now we show that $f(s, x, 1 / 2)>0, \forall s>x>0$. Substituting $\lambda=\frac{1}{2}$ gives

$$
f(s, x, 1 / 2)=\left(1+\frac{1}{2 x}+\frac{1}{2 s}\right) \ln \frac{s+1}{x+1}-\ln \frac{s}{x} .
$$

Because $\lim _{s \rightarrow x} f(s, x, 1 / 2)=0$ and

$$
\begin{aligned}
\frac{\partial f(s, x, 1 / 2)}{\partial s}=\frac{1}{2 s^{2}}\left[\frac{s(s-x)}{x(s+1)}-\ln \frac{s+1}{x+1}\right] \\
>\frac{1}{2 s^{2}}\left[\frac{s(s-x)}{x(s+1)}-\frac{s-x}{x+1}\right]=\frac{(s-x)^{2}}{2 x s^{2}(x+1)(s+1)}>0,
\end{aligned}
$$

we know that $f(s, x, 1 / 2)>0, \forall s>x>0$. Therefore, $f(s, x, \lambda)>0, \forall \lambda \geq 1 / 2, s>x>0$.

(ii). From $\lambda_{I} V_{I}>\lambda_{C} V_{C}$, we have $n_{C}=0$. With $n_{C}=0$, we know that

$$
\lambda_{I} V_{I}=\frac{\lambda_{I}(\gamma-1)\left(N-n_{I}\right)}{\rho+\lambda_{I} n_{I}} .
$$


Then $\lambda_{I} V_{I}=1$ and $N>\frac{\rho}{\lambda_{I}(\gamma-1)}$ implies $n_{I}>0$.

2. If $n_{I}>0$ and $n_{C}=0$, then $N>\frac{\rho}{\lambda_{I}(\gamma-1)}$ and $\lambda \geq 1 / 2$.

Since $n_{I}>0$ and $n_{C}=0$, we have

$$
\lambda_{I} V_{I}=\frac{\lambda_{I}(\gamma-1)\left(N-n_{I}\right)}{\rho+\lambda_{I} n_{I}} .
$$

Then $n_{I}=\frac{\lambda_{I}(\gamma-1) N-\rho}{\lambda_{I} \gamma}>0$ implies $N>\frac{\rho}{\lambda_{I}(\gamma-1)}$.

Also, $n_{C}=0$ implies $\lambda_{C} V_{C} \leq 1=\lambda_{I} V_{I}, \forall s>x>0$ (equality holds only if $n_{C}=0$ ). Thus we get

$$
\begin{aligned}
& \lim _{s \rightarrow x}\left(\lambda V_{I}-V_{C}\right)=\left[\frac{\lambda}{x+1}-\frac{1}{2(x+1)}\right]\left(N-n_{I}\right) \geq 0, \text { because } \\
& \lim _{s \rightarrow x} \frac{\lambda}{s-x} \ln \frac{s+1}{x+1}=\frac{\lambda}{x+1},
\end{aligned}
$$

and

$$
\lim _{s \rightarrow x} \frac{x}{(s-x)^{2}}\left[s \ln \frac{s}{x}-(s+1) \ln \frac{s+1}{x+1}\right]=\frac{1}{2(x+1)} .
$$

Therefore, $\lambda \geq 1 / 2$. Q.E.D.

\section{Appendix 7: Proof of Three Useful Inequalities}

In this appendix, we prove three inequalities which will be used in later proofs. The three inequities are

\section{Inequality 1:}

$$
\left(\ln \frac{s+1}{x+1}\right)\left[s \ln \frac{s}{x}-(s+1) \ln \frac{s+1}{x+1}\right]>\frac{s-x}{x+1}\left[(x+1) \ln \frac{s+1}{x+1}-x \ln \frac{s}{x}\right], \forall s>x>0 .(66)
$$




\section{Inequality 2:}

$$
\left(\ln \frac{s+1}{x+1}\right)\left[x \ln \frac{s}{x}-(x+1) \ln \frac{s+1}{x+1}\right]>\frac{s-x}{s+1}\left[(s+1) \ln \frac{s+1}{x+1}-s \ln \frac{s}{x}\right], \forall s>x>0 .(67)
$$

\section{Inequality 3:}

$$
\left(\ln \frac{s+1}{x+1}\right)\left[(x+s) \ln \frac{s}{x}-(x+s+1) \ln \frac{s+1}{x+1}\right]>\frac{s-x}{s+1}\left[(s+1) \ln \frac{s+1}{x+1}-\frac{x s}{x+1} \ln \frac{s}{x}\right]
$$

$\forall s>x>0$

Proof: We prove Inequality 1 first. Define

$$
f(s) \equiv\left(\ln \frac{s+1}{x+1}\right)\left[s \ln \frac{s}{x}-(s+1) \ln \frac{s+1}{x+1}\right]-\frac{s-x}{x+1}\left[(x+1) \ln \frac{s+1}{x+1}-x \ln \frac{s}{x}\right] .
$$

Then we have

$$
\begin{aligned}
g(x) \equiv & f^{\prime}(s) \\
= & \frac{1}{s+1}\left[s \ln \frac{s}{x}-(s+1) \ln \frac{s+1}{x+1}\right]+\left(\ln \frac{s+1}{x+1}\right)\left[\ln \frac{s}{x}-\ln \frac{s+1}{x+1}\right] \\
& -\frac{1}{x+1}\left[(x+1) \ln \frac{s+1}{x+1}-x \ln \frac{s}{x}\right]-\frac{s-x}{x+1}\left[\frac{x+1}{s+1}-\frac{x}{s}\right], \\
h(s) \equiv & g^{\prime}(x) \\
= & \frac{1}{s+1}\left[-\frac{s}{x}+\frac{s+1}{x+1}\right]-\frac{1}{x+1}\left[\ln \frac{s}{x}-\ln \frac{s+1}{x+1}\right] \\
& +\left(\ln \frac{s+1}{x+1}\right)\left[-\frac{1}{x}+\frac{1}{x+1}\right]+\frac{1}{(x+1)^{2}}\left[(x+1) \ln \frac{s+1}{x+1}-x \ln \frac{s}{x}\right] \\
& +\frac{1+s}{(x+1)^{2}}\left[\frac{x+1}{s+1}-\frac{x}{s}\right]-\frac{s-x}{x+1}\left[\frac{1}{s+1}-\frac{1}{s}\right], \\
h^{\prime}(s)= & -\frac{1}{(s+1)^{2}}\left[-\frac{s}{x}+\frac{s+1}{x+1}\right]+\frac{2}{s+1}\left[-\frac{1}{x}+\frac{1}{x+1}\right]+\frac{2}{(x+1)^{2}}\left[\frac{x+1}{s+1}-\frac{x}{s}\right] \\
& +\frac{1+s}{(x+1)^{2}}\left[-\frac{x+1}{(s+1)^{2}}+\frac{x}{s^{2}}\right]-\frac{s-x}{x+1}\left[-\frac{1}{(s+1)^{2}}+\frac{1}{s^{2}}\right]
\end{aligned}
$$




$$
=-\frac{x s(1+x s+2 s)+(s-x)\left(s^{2}+x^{2}+2 x^{2} s+3 x s+2 x+2 s\right)}{x s^{2}(x+1)^{2}(s+1)^{2}}<0 .
$$

Obviously, $f(s=x)=0, g(x=s)=0$ and $h(s=x)=0$. Then, $h^{\prime}(s)<0$ and $h(s=x)=0$ $\Rightarrow h(s)<0 \Rightarrow g^{\prime}(x)<0$ (along with $\left.g(x=s)=0\right) \Rightarrow g(x)>0 \Rightarrow f^{\prime}(s)>0$ (together with $f(s=x)=0) \Rightarrow f(s)>0$. Therefore, inequality 1 holds.

Inequality 2 is equivalent to

$$
\begin{aligned}
& \left(\ln \frac{s+1}{x+1}\right)\left[s \ln \frac{s}{x}-(s+1) \ln \frac{s+1}{x+1}\right] \\
& >\left[\frac{(x+1)(s+1)\left(\ln \frac{s+1}{x+1}\right)^{2}}{(s-x)^{2}}\right] \frac{s-x}{x+1}\left[(x+1) \ln \frac{s+1}{x+1}-x \ln \frac{s}{x}\right]
\end{aligned}
$$

If we can show that

$$
\frac{(x+1)(s+1)\left(\ln \frac{s+1}{x+1}\right)^{2}}{(s-x)^{2}}<1
$$

then (69) and Inequality 1 ensures that Inequality 2 is also true. But (69) is guaranteed by

$$
\ln \frac{s+1}{x+1}<\frac{s-x}{[(x+1)(s+1)]^{1 / 2}}
$$

We rewrite Inequality 3 as

$$
\begin{gathered}
\left(\ln \frac{s+1}{x+1}\right)\left[x \ln \frac{s}{x}-(x+1) \ln \frac{s+1}{x+1}\right]+s\left(\ln \frac{s+1}{x+1}\right)\left[\ln \frac{s}{x}-\ln \frac{s+1}{x+1}\right] \\
>\frac{s-x}{s+1}\left[(s+1) \ln \frac{s+1}{x+1}-s \ln \frac{s}{x}\right]+\frac{s(s-x)}{(s+1)(x+1)} \ln \frac{s}{x}
\end{gathered}
$$

Since inequality 2 holds, we just need to show that

$$
\left(\ln \frac{s+1}{x+1}\right)\left[\ln \frac{s}{x}-\ln \frac{s+1}{x+1}\right] \geq \frac{s-x}{(s+1)(x+1)} \ln \frac{s}{x} .
$$

To show (70), we define 


$$
f(s) \equiv\left(\ln \frac{s+1}{x+1}\right)\left[\ln \frac{s}{x}-\ln \frac{s+1}{x+1}\right]-\frac{s-x}{(s+1)(x+1)} \ln \frac{s}{x}
$$

Then we have

$$
\begin{aligned}
g(x) \equiv f^{\prime}(s) & \\
= & \frac{1}{s+1}\left[\ln \frac{s}{x}-\ln \frac{s+1}{x+1}\right]+\left(\ln \frac{s+1}{x+1}\right)\left[\frac{1}{s}-\frac{1}{s+1}\right] \\
& -\frac{1}{(s+1)^{2}} \ln \frac{s}{x}-\frac{s-x}{s(x+1)(s+1)} \\
g^{\prime}(x)= & -\frac{(s-x)^{2}}{x s(x+1)^{2}(s+1)^{2}}<0
\end{aligned}
$$

Moreover, we have $f(s=x)=0$ and $g(x=s)=0$. Then, $g^{\prime}(x)<0$ and $g(x=s)=0$ $\Rightarrow g(x)>0 \Rightarrow f^{\prime}(s)>0$. (along with $\left.f(s=x)=0\right) \Rightarrow f(s)>0$, Hence, inequality 3 also holds. Q.E.D.

\section{Appendix 8: Proof of Proposition 4}

This appendix proves the existence of an equilibrium with both innovation and imitation. First, we show that the function $n_{I}$ defined by (26) is increasing in $n_{C}$ with $n_{I}\left(n_{C}=0\right)=0$, while the function $n_{I}$ defined by (27) is decreasing in $n_{C}$. Then we prove the necessary and sufficient conditions of Proposition 4. Finally, we show that the equilibrium is unique.

Proof: 1. Show that the function $n_{I}$ defined by (26) has the following properties: $\frac{d \pi_{I}}{d n_{C}}>0$ and $n_{I}\left(n_{C}=0\right)=0$.

Using the notations introduced in Appendix 4, we reformulate (26) as

$$
\frac{\lambda}{s-x} \ln \frac{s+1}{x+1}=\frac{x}{(s-x)^{2}}\left[s \ln \frac{s}{x}-(s+1) \ln \frac{s+1}{x+1}\right],
$$


which can be simplified to

$$
1+\frac{\lambda}{x}+\frac{1-\lambda}{s}=\frac{\ln (s)-\ln (x)}{\ln (s+1)-\ln (x+1)} .
$$

Differentiating (71) with respect to $x$ gives

$$
\begin{aligned}
\frac{d s}{d x} & =\frac{-\frac{1}{x}+\frac{\lambda}{x^{2}} \ln \left(\frac{s+1}{x+1}\right)+\frac{1}{x+1}\left(1+\frac{1}{\lambda}+\frac{1-\lambda}{s}\right)}{-\frac{1}{s}-\frac{1-\lambda}{s^{2}} \ln \left(\frac{s+1}{x+1}\right)+\frac{1}{s+1}\left(1+\frac{1}{\lambda}+\frac{1-\lambda}{s}\right)} \\
& =\frac{\lambda \frac{s}{x} \ln \left(\frac{s+1}{x+1}\right)-(1-\lambda) \frac{s-x}{x+1}}{\lambda \frac{s-x}{s+1}-(1-\lambda) \frac{x}{s} \ln \left(\frac{s+1}{x+1}\right)} .
\end{aligned}
$$

Since $x=\lambda_{I} n_{I} / \rho, s=\left(\lambda_{I} n_{I}+\lambda_{C} n_{C}\right) / \rho, \frac{d n_{I}}{d n_{C}}>0$ is equivalent to $\frac{d s}{d x}>1$. Three sufficient conditions for $\frac{d s}{d x}>1$ are

Condition 1: $\lambda \frac{s}{x} \ln \left(\frac{s+1}{x+1}\right)-(1-\lambda) \frac{s-x}{x+1}>0$,

Condition 2: $\lambda \frac{s-x}{s+1}-(1-\lambda) \frac{x}{s} \ln \left(\frac{s+1}{x+1}\right)>0$,

Condition 3: $\lambda \frac{s}{x} \ln \left(\frac{s+1}{x+1}\right)-(1-\lambda) \frac{s-x}{x+1}>\lambda \frac{s-x}{s+1}-(1-\lambda) \frac{x}{s} \ln \left(\frac{s+1}{x+1}\right)$.

From (71), we have

$$
\lambda=\frac{x /(s-x)}{\ln \left(\frac{s+1}{x+1}\right)}\left[(s) \ln \left(\frac{s}{x}\right)-(s+1) \ln \left(\frac{s+1}{x+1}\right)\right] .
$$

Substituting $\lambda$ into the above three conditions, we can easily verify that the three conditions are equivalent to the three inequalities proven in Appendix 7. Therefore, $\frac{d n_{r}}{d n_{C}}>0$. Clearly, $n_{I}\left(n_{C}=0\right)=0$ and $n_{C}\left(n_{I}=0\right)=0$.

2. Show that the function $n_{I}$ defined by (27) has the property: $\frac{d n_{I}}{d n_{C}}<0$.

Differentiating equation (27), we have

$$
\frac{d n_{I}}{d n_{C}}=\frac{\ln \left(\frac{s+1}{x+1}\right)+\frac{\lambda_{X}(s-x)\left(N-n_{I}-n_{C}\right)}{\rho(x+1)(s+1)}}{\left.-\frac{1}{n_{C}} \ln \left(\frac{s+1}{x+1}\right)\right]\left(N-n_{I}-n_{C}\right)-\ln \left(\frac{s+1}{x+1}\right)+\frac{\lambda_{C}\left(N-n_{X}-n_{C}\right)}{\rho(s+1)}} .
$$


Since the numerator is positive, the sign of $\frac{d n_{I}}{d n_{C}}$ depends on the denominator. Let $\mathcal{D}$ denote the denominator, then

$$
\begin{aligned}
\mathcal{D}= & -\left[\frac{N-n_{I}}{n_{C}} \ln \left(\frac{s+1}{x+1}\right)-\frac{\lambda_{C}\left(N-n_{I}-n_{C}\right)}{\rho(s+1)}\right]<-\left[\frac{N-n_{I}}{n_{C}} \frac{s-x}{s+1}-\frac{\lambda_{C}\left(N-n_{I}-n_{C}\right)}{\rho(s+1)}\right] \\
& \quad\left(\text { becauseln } \frac{s+1}{x+1}>\frac{s-x}{s+1}\right) \\
= & -\frac{s-x}{s+1}<0 .
\end{aligned}
$$

Hence, $\frac{d n_{I}}{d n_{C}}<0$.

3. Show that $n_{I}>0$ and $n_{C}>0$ if and only if $N>\frac{\rho}{\lambda_{I}(\gamma-1)}$ and $0<\lambda<\frac{1}{2}$.

(i) If $N>\frac{\rho}{\lambda_{I}(\gamma-1)}$ and $0<\lambda<\frac{1}{2}$, then $n_{I}>0$ and $n_{C}>0$.

First, we consider the condition $0<\lambda<\frac{1}{2}$. If $0<\lambda<\frac{1}{2}$, then there always exists a pair of $n_{I}$ and $n_{C}$ with $n_{I}>0$ and $n_{C}>0$ (or equivalently $0<x<s$ ) satisfying equation (26). This is because

$$
\begin{aligned}
& \lim _{x \rightarrow 0} \frac{\lambda}{s-x} \ln \frac{s+1}{x+1}=\frac{\lambda}{s} \ln (s+1) \\
& >\lim _{x \rightarrow 0} \frac{x}{(s-x)^{2}}\left[s \ln \frac{s}{x}-(s+1) \ln \frac{s+1}{x+1}\right]=0
\end{aligned}
$$

and

$$
\begin{aligned}
& \lim _{x \rightarrow s} \frac{\lambda}{s-x} \ln \frac{s+1}{x+1}=\frac{\lambda}{s+1} \\
& <\lim _{x \rightarrow s} \frac{x}{(s-x)^{2}}\left[s \ln \frac{s}{x}-(s+1) \ln \frac{s+1}{x+1}\right]=\frac{1}{2(s+1)}, \text { if } 0<\lambda<\frac{1}{2} .
\end{aligned}
$$


The condition $N>\frac{\rho}{\lambda_{I}(\gamma-1)}$ guarantees that the function defined by (27) has the properties: $n_{I}\left(n_{C}=0\right)>0$ and $n_{C}\left(n_{I}=0\right)>0$. This is because if $n_{I}=0$, then equation (27) becomes

$$
\frac{\lambda_{I}}{\lambda_{C} n_{C}}\left[\ln \left(\frac{\rho+\lambda_{C} n_{C}}{\rho}\right)\right](\gamma-1)\left(N-n_{C}\right)=1 \text {. }
$$

Since $\lim _{n_{C} \rightarrow 0} L H S=\frac{\lambda_{I}(\gamma-1) N}{\rho}$ and $\frac{\partial L H S}{\partial n_{C}}<0$, if $N>\frac{\rho}{\lambda_{I}(\gamma-1)}$, then $\frac{\lambda_{I}(\gamma-1) N}{\rho}>1$. Thus, $n_{C}>0$.

Also, if $n_{C}=0$, then equation (27) gives

$$
n_{I}=\frac{\lambda_{I}(\gamma-1) N-\rho}{\lambda_{I} \gamma}
$$

Then, $N>\frac{\rho}{\lambda_{I}(\gamma-1)}$ implies that $n_{I}>0$.

(ii) If $n_{I}>0$ and $n_{C}>0$, then $N>\frac{\rho}{\lambda_{I}(\gamma-1)}$ and $0<\lambda<\frac{1}{2}$.

From Appendix 4, we know that if $n_{C}>0$, then $0<\lambda<\frac{1}{2}$. We also know from (74) and (75) that if $n_{I}>0$ and $n_{C}>0$, then $N>\frac{\rho}{\lambda_{I}(\gamma-1)}$.

The uniqueness of the equilibrium is guaranteed by the monotonicity of the functions defined by (26) and (27). Q.E.D.

\section{Appendix 9: Proof of Propositions 5-8}

We prove the propositions in the comparative steady-state analysis by determining the signs of the derivatives of $n_{I}$ and $n_{C}$ with respect to the model's parameters (i.e. $\lambda_{I}, \lambda_{C}, \gamma, \rho$ and $N)$. The equilibrium conditions for the model are

$$
1+\frac{\lambda}{x}+\frac{1-\lambda}{s}=\frac{\ln (s)-\ln (x)}{\ln (s+1)-\ln (x+1)},
$$




$$
\frac{\lambda}{n_{C}}\left[\ln \left(\frac{s+1}{x+1}\right)\right](\gamma-1)\left(N-n_{I}-n_{C}\right)=1
$$

Differentiating these two equations with respect to parameter $i\left(i=\lambda_{I}, \lambda_{C}, \gamma, \rho\right.$ and $\left.N\right)$ gives

$$
\left[\begin{array}{ll}
a_{11}^{i} & a_{12}^{i} \\
a_{21}^{i} & a_{22}^{i}
\end{array}\right]\left[\begin{array}{l}
\partial n_{I} / \partial i \\
\partial n_{C} / \partial i
\end{array}\right]=\left[\begin{array}{c}
c_{1}^{i} \\
c_{2}^{i}
\end{array}\right],
$$

where (the sign of each coefficient is explained at the end of this appendix)

$$
\begin{aligned}
& a_{11}^{i}=\lambda_{I}(a-b) / \rho<0 \\
& a_{12}^{i}=\lambda_{C} a / \rho>0, \\
& a_{21}^{i}=c<0 \\
& a_{22}^{i}=d<0 \\
& c_{1}^{\lambda_{I}}=\frac{1}{\lambda_{I} s}\left[\left(\lambda+\frac{(1-\lambda) x}{s}\right) \ln \left(\frac{s+1}{x+1}\right)-\frac{(1+\lambda x+(1-\lambda) s)(s-x)}{(s+1)(x+1)}\right]<0 \\
& c_{2}^{\lambda_{I}}=\frac{n_{I}}{\rho}\left[\frac{s-x}{(s+1)(x+1)}-\frac{1}{x} \ln \left(\frac{s+1}{x+1}\right)\right]\left(N-n_{I}-n_{C}\right)<0, \\
& c_{1}^{\lambda_{c}}=\frac{s-x}{\lambda_{C} s}\left[\left(\frac{\lambda}{x}+\frac{1-\lambda}{s}\right) \ln \frac{s+1}{x+1}-\frac{\lambda(s-x)}{x(s+1)}\right]>0, \\
& c_{2}^{\lambda_{c}}=\frac{n_{C}}{\rho}\left[\frac{1}{s-x} \ln \left(\frac{s+1}{x+1}\right)-\frac{1}{s+1}\right]\left(N-n_{I}-n_{C}\right)>0, \\
& c_{1}^{\gamma}=0 \\
& c_{2}^{\gamma}=-\frac{1}{\gamma-1}\left[\ln \left(\frac{s+1}{x+1}\right)\right]\left(N-n_{I}-n_{C}\right)<0, \\
& c_{1}^{\rho}=\frac{1}{\rho}\left[-\left(\frac{\lambda}{x}+\frac{1-\lambda}{s}\right) \ln \left(\frac{s+1}{x+1}\right)+\left(1+\frac{\lambda}{x}+\frac{1-\lambda}{s}\right) \frac{s-x}{(s+1)(x+1)}\right]<0, \\
& c_{2}^{\rho}=\frac{(s-x)\left(N-n_{I}-n_{C}\right)}{\rho(s+1)(x+1)}>0, \\
& c_{1}^{N}=0,
\end{aligned}
$$




$$
c_{2}^{N}=-\ln \left(\frac{s+1}{x+1}\right)<0
$$

and where

$$
\begin{aligned}
& a=-\frac{1}{s}-\frac{1-\lambda}{s^{2}} \ln \left(\frac{s+1}{x+1}\right)+\frac{1}{s+1}\left(1+\frac{\lambda}{x}+\frac{1-\lambda}{s}\right)>0, \\
& b=-\frac{1}{x}+\frac{\lambda}{x^{2}} \ln \left(\frac{s+1}{x+1}\right)+\frac{1}{x+1}\left(1+\frac{\lambda}{x}+\frac{1-\lambda}{s}\right)>0, \\
& c=-\frac{\lambda_{I}(s-x)\left(N-n_{I}-n_{C}\right)}{\rho(s+1)(x+1)}-\ln \left(\frac{s+1}{x+1}\right)<0, \\
& d=-\frac{1}{n_{C}}\left(\ln \left(\frac{s+1}{x+1}\right)-\frac{s-x}{s+1}\right)\left(N-n_{I}-n_{C}\right)-\ln \left(\frac{s+1}{x+1}\right)<0 .
\end{aligned}
$$

Then

$$
\begin{aligned}
& D^{i}=\left|\begin{array}{ll}
a_{11}^{i} & a_{12}^{i} \\
a_{21}^{i} & a_{22}^{i}
\end{array}\right|=a_{11}^{i} a_{22}^{i}-a_{12}^{i} a_{21}^{i}>0, \\
& D_{I}^{\lambda_{I}}=\left|\begin{array}{ll}
c_{1}^{\lambda_{I}} & a_{12}^{\lambda_{I}} \\
c_{2}^{\lambda_{I}} & a_{22}^{\lambda_{I}}
\end{array}\right|=c_{1}^{\lambda_{I}} a_{22}^{\lambda_{I}}-a_{12}^{\lambda_{I}} c_{2}^{\lambda_{I}}>0, \\
& D_{C}^{\lambda_{I}}=\left|\begin{array}{ll}
a_{11}^{\lambda_{I}} & c_{1}^{\lambda_{I}} \\
a_{21}^{\lambda_{I}} & c_{2}^{\lambda_{I}}
\end{array}\right|=c_{2}^{\lambda_{I}} a_{11}^{\lambda_{I}}-a_{21}^{\lambda_{I}} c_{1}^{\lambda_{I}}, \\
& D_{I}^{\lambda_{c}}=\left|\begin{array}{ll}
c_{1}^{\lambda_{c}} & a_{12}^{\lambda_{c}} \\
c_{2}^{\lambda_{C}} & a_{22}^{\lambda_{c}}
\end{array}\right|=c_{1}^{\lambda_{c}} a_{22}^{\lambda_{C}}-a_{12}^{\lambda_{C}} c_{2}^{\lambda_{c}}<0, \\
& D_{C}^{\lambda_{c}}=\left|\begin{array}{ll}
a_{11}^{\lambda_{c}} & c_{1}^{\lambda_{c}} \\
a_{21}^{\lambda_{C}} & c_{2}^{\lambda_{c}}
\end{array}\right|=c_{2}^{\lambda_{c}} a_{11}^{\lambda_{C}}-a_{21}^{\lambda_{C}} c_{1}^{\lambda_{C}}, \\
& D_{I}^{\gamma}=\left|\begin{array}{ll}
c_{1}^{\gamma} & a_{12}^{\gamma} \\
c_{2}^{\gamma} & a_{22}^{\gamma}
\end{array}\right|=-c_{2}^{\gamma} a_{12}^{\gamma}>0, \\
& D_{C}^{\gamma}=\left|\begin{array}{ll}
a_{11}^{\gamma} & c_{1}^{\gamma} \\
a_{21}^{\gamma} & c_{2}^{\gamma}
\end{array}\right|=c_{2}^{\gamma} a_{11}^{\gamma}>0, \\
& D_{I}^{\rho}=\left|\begin{array}{ll}
c_{1}^{\rho} & a_{12}^{\rho} \\
c_{2}^{\rho} & a_{22}^{\rho}
\end{array}\right|=c_{1}^{\rho} a_{22}^{\rho}-c_{2}^{\rho} a_{12}^{\rho},
\end{aligned}
$$




$$
\begin{aligned}
& D_{C}^{\rho}=\left|\begin{array}{ll}
a_{11}^{\rho} & c_{1}^{\rho} \\
a_{21}^{\rho} & c_{2}^{\rho}
\end{array}\right|=c_{2}^{\rho} a_{11}^{\rho}-c_{1}^{\rho} a_{21}^{\rho}<0, \\
& D_{I}^{N}=\left|\begin{array}{ll}
c_{1}^{N} & a_{12}^{N} \\
c_{2}^{N} & a_{22}^{N}
\end{array}\right|=-c_{2}^{N} a_{12}^{N}>0, \\
& D_{C}^{N}=\left|\begin{array}{ll}
a_{11}^{N} & c_{1}^{N} \\
a_{21}^{N} & c_{2}^{N}
\end{array}\right|=c_{2}^{N} a_{11}^{N}>0 .
\end{aligned}
$$

Therefore, we have

$$
\begin{aligned}
& \frac{\partial n_{I}}{\partial \lambda_{I}}=\frac{D_{I}^{\lambda_{I}}}{D^{\lambda_{I}}}>0, \quad \frac{\partial n_{C}}{\partial \lambda_{I}}=\frac{D_{C}^{\lambda_{I}}}{D^{\lambda_{I}}}(\text { see Table } 4) \\
& \frac{\partial n_{I}}{\partial \lambda_{C}}=\frac{D_{I}^{\lambda_{C}}}{D^{\lambda_{C}}}<0, \quad \frac{\partial n_{C}}{\partial \lambda_{C}}=\frac{D_{C}^{\lambda_{C}}}{D^{\lambda_{C}}}(\text { see Table 5); } \\
& \frac{\partial n_{I}}{\partial \gamma}=\frac{D_{I}^{\gamma}}{D^{\gamma}}>0, \quad \frac{\partial n_{C}}{\partial \gamma}=\frac{D_{C}^{\gamma}}{D^{\gamma}}>0 \\
& \frac{\partial n_{I}}{\partial \rho}=\frac{D_{I}^{\rho}}{D^{\rho}}(\text { see Table } 6), \quad \frac{\partial n_{C}}{\partial \rho}=\frac{D_{C}^{\rho}}{D^{\rho}}<0 \\
& \frac{\partial n_{I}}{\partial N}=\frac{D_{I}^{N}}{D^{N}}>0, \quad \frac{\partial n_{C}}{\partial N}=\frac{D_{C}^{N}}{D^{N}}>0 .
\end{aligned}
$$

\section{Explanations of the signs of the above coefficients:}

- The sign of $a_{11}^{i}$ is determined by the size of $(a-b)$. It is easily verified that $a-b<0$ is equivalent to Inequality 3 in Appendix 7.

- $a_{12}^{i}$ and $a$ has the same sign. It is shown that $a>0$ is equivalent to Inequality 2 in Appendix 7.

- The sign of $a_{21}^{i}$ (i.e. c) is obvious.

- The sign of $a_{22}^{i}$ (i.e. $d$ ) is guaranteed by 


$$
\ln \frac{s+1}{x+1}>\frac{s-x}{s+1}
$$

- $c_{1}^{\lambda_{I}}<0$ because

$$
\begin{aligned}
c_{1}^{\lambda_{I}}= & \frac{1}{\lambda_{I} s}\left[\left(\lambda+\frac{(1-\lambda) x}{s}\right) \ln \left(\frac{s+1}{x+1}\right)-\frac{(1+\lambda x+(1-\lambda) s)(s-x)}{(s+1)(x+1)}\right] \\
< & \frac{1}{\lambda_{I} s}\left[\left(\frac{\lambda s+(1-\lambda) x}{s}\right) \frac{s-x}{x+1}-\frac{(1+\lambda x+(1-\lambda) s)(s-x)}{(s+1)(x+1)}\right] \\
& \left.\quad \text { (because } \ln \frac{s+1}{x+1}<\frac{s-x}{x+1}\right) \\
= & -\frac{(s-x)^{2}}{\lambda_{I} s^{2}(x+1)(s+1)}[(1-\lambda)+(1-2 \lambda) s]<0, \text { if } \lambda<\frac{1}{2} .
\end{aligned}
$$

- $c_{2}^{\lambda_{I}}<0$ because

$$
\begin{aligned}
c_{2}^{\lambda_{I}} & =\frac{n_{I}}{\rho}\left[\frac{s-x}{(s+1)(x+1)}-\frac{1}{x} \ln \left(\frac{s+1}{x+1}\right)\right]\left(N-n_{I}-n_{C}\right) \\
& <\frac{n_{I}}{\rho}\left[\frac{s-x}{(s+1)(x+1)}-\frac{1}{x} \frac{s-x}{s+1}\right]\left(N-n_{I}-n_{C}\right) \\
& =\frac{n_{I}(s-x)}{\rho(s+1)}\left[\frac{1}{x+1}-\frac{1}{x}\right]\left(N-n_{I}-n_{C}\right)<0 .
\end{aligned}
$$

- $c_{1}^{\lambda c}>0$ is proven as follows:

Substituting $\lambda$ into this inequality, we have

$$
\frac{s-x}{\lambda_{C} s}\left(\ln \frac{s+1}{x+1}\right)^{-1}\left[\ln \frac{s+1}{x+1}\left(\ln \frac{s}{x}-\ln \frac{s+1}{x+1}\right)-\left(\frac{s}{s+1} \ln \frac{s}{x}-\ln \frac{s+1}{x+1}\right)\right]>0
$$

Define

$$
f(x) \equiv \ln \frac{s+1}{x+1}\left(\ln \frac{s}{x}-\ln \frac{s+1}{x+1}\right)-\left(\frac{s}{s+1} \ln \frac{s}{x}-\ln \frac{s+1}{x+1}\right)>0
$$


then we need to show that $f(x)>0$. Two sufficient conditions for $f(x)>0$ are $f(x=s)=0$ and $f^{\prime}(x)<0$. Let

$$
\begin{aligned}
g(s) \equiv & f^{\prime}(x)=-\frac{1}{x+1}\left[\ln \frac{s}{x}-\ln \frac{s+1}{x+1}\right] \\
& +\ln \frac{s+1}{x+1}\left[-\frac{1}{x}+\frac{1}{x+1}\right]+\frac{s}{x(s+1)}-\frac{1}{x+1} .
\end{aligned}
$$

Now we need to show $g(s)<0$, whose sufficient conditions are $g(s=x)=0$ and $g^{\prime}(s)<0$.

$$
g^{\prime}(s)=-\left[\frac{1}{s(x+1)(s+1)}+\frac{1}{x(x+1)(s+1)}+\frac{1}{x(s+1)^{2}}\right]<0 .
$$

So $g^{\prime}(s)<0$ and $g(s=x)=0 \Rightarrow g(s)<0 \Rightarrow f^{\prime}(x)<0$ ( along with $f(x=s)=0$ )

$\Rightarrow f(x)>0$.

- $c_{2}^{\lambda_{c}}>0$ because

$$
\begin{aligned}
c_{2}^{\lambda_{C}}= & \frac{n_{C}}{\rho}\left[\frac{1}{s-x} \ln \left(\frac{s+1}{x+1}\right)-\frac{1}{s+1}\right]\left(N-n_{I}-n_{C}\right)>0 \\
= & \frac{n_{C}}{\rho(s-x)}\left[\ln \left(\frac{s+1}{x+1}\right)-\frac{s-x}{s+1}\right]\left(N-n_{I}-n_{C}\right)>0 \\
& \left.\quad \text { (again because } \ln \frac{s+1}{x+1}>\frac{s-x}{s+1}\right) .
\end{aligned}
$$

- $c_{2}^{\gamma}<0$ is obvious.

- $c_{1}^{\rho}<0$ because

$$
\begin{aligned}
c_{1}^{\rho} & =\frac{1}{\rho}\left[-\left(\frac{\lambda}{x}+\frac{1-\lambda}{s}\right) \ln \left(\frac{s+1}{x+1}\right)+\left(1+\frac{\lambda}{x}+\frac{1-\lambda}{s}\right) \frac{s-x}{(s+1)(x+1)}\right] \\
& =-\frac{1}{\rho}\left[\left(\ln \frac{s}{x}-\ln \frac{s+1}{x+1}\right)-\frac{\ln (s / x)}{\ln ((s+1) /(x+1))} \frac{s-x}{(x+1)(s+1)}\right]
\end{aligned}
$$


Table 4: The Effect of $\lambda_{I}$ on $n_{C}$

\begin{tabular}{llll}
\hline \hline$\lambda_{I}$ & $\theta_{1}$ & $n_{I}$ & $n_{C}$ \\
\hline 0.1 & 0.004690 & 0.005263 & 0.037228 \\
0.2 & 0.007329 & 0.013136 & 0.118613 \\
0.3 & 0.010861 & 0.020882 & 0.190179 \\
0.4 & 0.015769 & 0.029701 & 0.247179 \\
0.5 & 0.022747 & 0.040440 & 0.289560 \\
0.6 & 0.032864 & 0.054025 & 0.317979 \\
0.7 & 0.047741 & 0.071538 & 0.332952 \\
0.8 & 0.069796 & 0.094198 & 0.334777 \\
0.9 & 0.102510 & 0.123238 & 0.323689 \\
1.0 & 0.150773 & 0.159777 & 0.299981 \\
1.1 & 0.221401 & 0.204738 & 0.264000 \\
1.2 & 0.324015 & 0.258876 & 0.216035 \\
1.3 & 0.472500 & 0.322868 & 0.156196 \\
1.4 & 0.687409 & 0.397415 & 0.084336 \\
\hline
\end{tabular}

Note: In Tables 47, the model's parameters

which are not shown in the tables are:

$\left(\lambda_{I}, \lambda_{C}, \gamma, \rho, N\right)=(1,3,2,0.05,1)$.

(because of (76))

$=-\frac{1}{\rho \ln ((s+1) /(x+1))}\left[\left(\ln \frac{s+1}{x+1}\right)\left(\ln \frac{s}{x}-\ln \frac{s+1}{x+1}\right)-\frac{s-x}{(x+1)(s+1)} \ln \frac{s}{x}\right]<0$

(see proof of Inequality 3 in Appendix 7).

- $c_{2}^{\rho}>0$ and $c_{1}^{N}<0$ are obvious. Q.E.D.

\section{Appendix 10: Proof of Proposition 9}

Proof: With a subsidy $s_{I}$ to innovative $\mathrm{R} \& \mathrm{D}$, the equilibrium conditions become

$$
\begin{aligned}
& 1+\frac{\lambda}{x\left(1-s_{I}\right)}+\frac{1-s_{I}-\lambda}{s\left(1-s_{I}\right)}=\frac{\ln (s)-\ln (x)}{\ln (s+1)-\ln (x+1)}, \\
& \frac{\lambda}{n_{C}}\left[\ln \left(\frac{s+1}{x+1}\right)\right](\gamma-1)\left(N-n_{I}-n_{C}\right)=1-s_{I} .
\end{aligned}
$$


Table 5: The Effect of $\lambda_{C}$ on $n_{C}$

\begin{tabular}{clll}
\hline \hline$\lambda_{C}$ & $\theta_{1}$ & $n_{I}$ & $n_{C}$ \\
\hline 2.1 & 0.770232 & 0.415692 & 0.059050 \\
2.5 & 0.329576 & 0.259223 & 0.210925 \\
3.0 & 0.150773 & 0.159777 & 0.299981 \\
3.5 & 0.083149 & 0.107584 & 0.338941 \\
4.0 & 0.051841 & 0.077494 & 0.354339 \\
4.5 & 0.035231 & 0.058798 & 0.357810 \\
5.0 & 0.025498 & 0.046444 & 0.355002 \\
5.5 & 0.019350 & 0.037858 & 0.348846 \\
6.0 & 0.015230 & 0.031637 & 0.340934 \\
6.5 & 0.012338 & 0.026972 & 0.332165 \\
7.0 & 0.010137 & 0.023373 & 0.326060 \\
7.5 & 0.008644 & 0.020529 & 0.313922 \\
8.0 & 0.007420 & 0.018235 & 0.304928 \\
8.5 & 0.006454 & 0.016353 & 0.296181 \\
9.0 & 0.005677 & 0.014786 & 0.287739 \\
9.5 & 0.005043 & 0.013465 & 0.279628 \\
10.0 & 0.004518 & 0.012338 & 0.271860 \\
\hline
\end{tabular}

Table 6: The Effect of $\rho$ on $n_{I}$

\begin{tabular}{clll}
\hline \hline$\rho$ & $\theta_{1}$ & $n_{I}$ & $n_{C}$ \\
\hline 0.02 & 0.131826 & 0.150900 & 0.331265 \\
0.04 & 0.144846 & 0.157289 & 0.309538 \\
0.06 & 0.156388 & 0.161891 & 0.291100 \\
0.08 & 0.166838 & 0.165187 & 0.274973 \\
0.10 & 0.176431 & 0.167469 & 0.260578 \\
0.12 & 0.185323 & 0.168931 & 0.247539 \\
0.14 & 0.193626 & 0.169711 & 0.235593 \\
0.16 & 0.201420 & 0.169911 & 0.224551 \\
0.18 & 0.208769 & 0.169609 & 0.214272 \\
0.20 & 0.215723 & 0.168869 & 0.204645 \\
0.22 & 0.222323 & 0.167740 & 0.195583 \\
0.24 & 0.228600 & 0.166262 & 0.187014 \\
0.26 & 0.234587 & 0.164473 & 0.178881 \\
0.28 & 0.240305 & 0.162399 & 0.171135 \\
0.30 & 0.245776 & 0.160068 & 0.163736 \\
\hline
\end{tabular}


Differentiating (80) and (81) with respect to $s_{I}$ gives

$$
\left[\begin{array}{ll}
a_{11}^{s_{I}} & a_{12}^{s_{I}} \\
a_{21}^{s_{I}} & a_{22}^{s_{I}}
\end{array}\right]\left[\begin{array}{l}
\partial n_{I} / \partial s_{I} \\
\partial n_{C} / \partial s_{I}
\end{array}\right]=\left[\begin{array}{c}
c_{1}^{s_{I}} \\
c_{2}^{s_{I}}
\end{array}\right],
$$

where

$$
\begin{aligned}
& a_{11}^{s_{I}}=\lambda_{I}\left(a^{s_{I}}-b^{s_{I}}\right) / \rho<0, \\
& a_{12}^{s_{I}}=\lambda_{C} a^{s_{I}} / \rho>0, \\
& c_{1}^{s_{I}}=-\frac{\lambda(s-x)}{x s\left(1-s_{I}\right)^{2}} \ln \left(\frac{s+1}{x+1}\right)<0, \\
& a_{21}^{s_{I}}=c<0 \\
& a_{22}^{s_{I}}=d<0 \\
& c_{2}^{s_{I}}=-\frac{n_{C}}{\lambda(\gamma-1)}<0,
\end{aligned}
$$

and where

$$
\begin{aligned}
& a^{s_{I}}=-\frac{1}{s}-\frac{1-s_{I}-\lambda}{s^{2}\left(1-s_{I}\right)} \ln \left(\frac{s+1}{x+1}\right)+\frac{1}{s+1}\left(1+\frac{\lambda}{x\left(1-s_{I}\right)}+\frac{1-s_{I}-\lambda}{s\left(1-s_{I}\right)}\right)>0 \\
& b^{s_{I}}=-\frac{1}{x}+\frac{\lambda}{x^{2}\left(1-s_{I}\right)} \ln \left(\frac{s+1}{x+1}\right)+\frac{1}{x+1}\left(1+\frac{\lambda}{x\left(1-s_{I}\right)}+\frac{1-s_{I}-\lambda}{s\left(1-s_{I}\right)}\right)>0 .
\end{aligned}
$$

Note that we consider only small subsidies, so the signs of the above coefficients are guaranteed by Appendix 9 and by using continuity arguments. Then

$$
\begin{aligned}
& D^{s_{I}}=\left|\begin{array}{ll}
a_{11}^{s_{I}} & a_{12}^{s_{I}} \\
a_{21}^{s_{I}} & a_{22}^{s_{I}}
\end{array}\right|=a_{11}^{s_{I}} a_{22}^{s_{I}}-a_{12}^{s_{I}} a_{21}^{s_{I}}>0, \\
& D_{I}^{s_{I}}=\left|\begin{array}{ll}
c_{1}^{s_{I}} & a_{12}^{s_{I}} \\
c_{2}^{s_{I}} & a_{22}^{s_{I}}
\end{array}\right|=c_{1}^{s_{I}} a_{22}^{s_{I}}-a_{12}^{s_{I}} c_{2}^{s_{I}}>0, \\
& D_{C}^{s_{I}}=\left|\begin{array}{ll}
a_{11}^{s_{I}} & c_{1}^{s_{I}} \\
a_{21}^{s_{I}} & c_{2}^{s_{I}}
\end{array}\right|=c_{2}^{s_{I}} a_{11}^{s_{I}}-a_{21}^{s_{I}} c_{1}^{s_{I}} .
\end{aligned}
$$

So we have 
Table 7: The Effect of $s_{I}$ on $n_{C}$

\begin{tabular}{clll}
\hline \hline \multicolumn{4}{l}{$\lambda_{C}=2.5$} \\
\hline \hline$s_{I}$ & $\theta_{1}$ & $n_{I}$ & $n_{C}$ \\
0.10 & 0.540869 & 0.369270 & 0.125386 \\
0.11 & 0.571670 & 0.382967 & 0.114777 \\
0.12 & 0.604959 & 0.397225 & 0.103756 \\
0.13 & 0.640994 & 0.412060 & 0.092314 \\
0.14 & 0.680061 & 0.427489 & 0.080446 \\
0.15 & 0.722490 & 0.443528 & 0.068144 \\
0.16 & 0.768656 & 0.460191 & 0.055402 \\
0.17 & 0.818974 & 0.477492 & 0.042218 \\
0.18 & 0.873924 & 0.495443 & 0.028590 \\
0.19 & 0.934059 & 0.514054 & 0.014516 \\
\hline \hline & $\lambda_{C}=6$ & & \\
\hline \hline$s_{I}$ & $\theta_{1}$ & $n_{I}$ & $n_{C}$ \\
0.10 & 0.019357 & 0.042085 & 0.355341 \\
0.11 & 0.019890 & 0.043421 & 0.356612 \\
0.12 & 0.020450 & 0.044824 & 0.357840 \\
0.13 & 0.021041 & 0.046299 & 0.359020 \\
0.14 & 0.021664 & 0.047851 & 0.360148 \\
0.15 & 0.022322 & 0.049484 & 0.361219 \\
0.16 & 0.023018 & 0.051205 & 0.362229 \\
0.17 & 0.023754 & 0.053019 & 0.363171 \\
0.18 & 0.024533 & 0.054934 & 0.364040 \\
0.19 & 0.026019 & 0.056957 & 0.364830 \\
0.20 & 0.026945 & 0.059096 & 0.365533 \\
\hline & & &
\end{tabular}




$$
\frac{\partial n_{I}}{\partial s_{I}}=\frac{D_{I}^{s_{I}}}{D^{s_{I}}}>0, \quad \frac{\partial n_{C}}{\partial s_{I}}=\frac{D_{C}^{s_{I}}}{D^{s_{I}}}(\text { see Table } 7)
$$

Q.E.D.

\section{Appendix 11: Proof of Proposition 10}

Proof: Let $s_{C}$ be a subsidy to imitative $R \& D$, then the equilibrium conditions are

$$
\begin{aligned}
& 1+\frac{\lambda\left(1-s_{C}\right)}{x}+\frac{1-\lambda\left(1-s_{C}\right)}{s}=\frac{\ln (s)-\ln (x)}{\ln (s+1)-\ln (x+1)}, \\
& \frac{\lambda}{n_{C}}\left[\ln \left(\frac{s+1}{x+1}\right)\right](\gamma-1)\left(N-n_{I}-n_{C}\right)=1 .
\end{aligned}
$$

Differentiating (83) and (84) with respect to $s_{C}$, we have

$$
\left[\begin{array}{ll}
a_{11}^{s c} & a_{12}^{s c} \\
a_{21}^{s c} & a_{22}^{s c}
\end{array}\right]\left[\begin{array}{l}
\partial n_{I} / \partial s_{C} \\
\partial n_{C} / \partial s_{C}
\end{array}\right]=\left[\begin{array}{l}
c_{1}^{s c} \\
c_{2}^{s c}
\end{array}\right]
$$

where

$$
\begin{aligned}
& a_{11}^{s c}=\lambda_{I}\left(a^{s c}-b^{s c}\right) / \rho<0, \\
& a_{12}^{s c}=\lambda_{C} a^{s c} / \rho>0, \\
& c_{1}^{s c}=\frac{\lambda(s-x)}{x s} \ln \left(\frac{s+1}{x+1}\right)>0, \\
& a_{21}^{s c}=c<0, \\
& a_{22}^{s c}=d<0, \\
& c_{2}^{s c}=0,
\end{aligned}
$$

and where

$$
a^{s^{c}}=-\frac{1}{s}-\frac{1-\lambda\left(1-s_{C}\right)}{s^{2}} \ln \left(\frac{s+1}{x+1}\right)+\frac{1}{s+1}\left(1+\frac{\lambda\left(1-s_{C}\right)}{x}+\frac{1-\lambda\left(1-s_{C}\right)}{s}\right)
$$




$$
\begin{aligned}
>0 \\
b^{s c}=-\frac{1}{x}+\frac{\lambda\left(1-s_{C}\right)}{x^{2}} \ln \left(\frac{s+1}{x+1}\right)+\frac{1}{x+1}\left(1+\frac{\lambda\left(1-s_{C}\right)}{x}+\frac{1-\lambda\left(1-s_{C}\right)}{s}\right)>0 .
\end{aligned}
$$

Note that, as in Appendix 10, we consider only small subsidies, so by continuity considerations, the signs of the above coefficients are guaranteed by Appendix 9. Then

$$
\begin{aligned}
& D^{s_{c}}=\left|\begin{array}{ll}
a_{11}^{s c} & a_{12}^{s c} \\
a_{21}^{s c} & a_{22}^{s c}
\end{array}\right|=a_{11}^{s c} a_{22}^{s c}-a_{12}^{s c} a_{21}^{s c}>0, \\
& D_{I}^{s c}=\left|\begin{array}{ll}
c_{1}^{s c} & a_{12}^{s c} \\
c_{2}^{s c} & a_{22}^{s c}
\end{array}\right|=c_{1}^{s c} a_{22}^{s c}<0, \\
& D_{C}^{s c}=\left|\begin{array}{ll}
a_{11}^{s c} & c_{1}^{s c} \\
a_{21}^{s c} & c_{2}^{s c}
\end{array}\right|=-a_{21}^{s c} c_{1}^{s c}>0 .
\end{aligned}
$$

So we have

$$
\frac{\partial n_{I}}{\partial s_{C}}=\frac{D_{I}^{s c}}{D^{s c}}<0, \quad \frac{\partial n_{C}}{\partial s_{C}}=\frac{D_{C}^{s c}}{D^{s c}}>0
$$

Q.E.D.

\section{Appendix 12: Derivation of $V_{I}$ and $V_{C}$ with $\Omega^{\prime}(M)>0$}

To derive the value functions of innovation and imitation, we need to know the profit flows for innovators and imitators. Since $p\left(M_{-1}, M\right)=\beta \Omega(M)$ and the profit flow for an innovator or imitator is $\pi\left(M_{-1}, M\right)=\left(\frac{p-1}{p}\right) \frac{E}{M}$, with the assumption (34), we have

$$
p\left(M \mid \text { conditional on } M_{-1}\right)=\left\{\begin{array}{l}
\beta, M=1, \text { with probability } \theta_{1}, \\
\beta \nu, M \geq 2, \text { with probability }\left(1-\theta_{1}\right),
\end{array}\right.
$$

and

$$
\pi\left(M \mid \text { conditional on } M_{-1}\right)=\left\{\begin{array}{l}
\left(\frac{\beta-1}{\beta}\right) E, M=1, \text { with probability } \theta_{1}, \\
\left(\frac{\beta \nu-1}{\beta \nu}\right) \frac{E}{M}, M \geq 2, \text { with probability }\left(1-\theta_{1}\right) .
\end{array}\right.
$$

Then the stationary distribution of the type of sector and $\beta=\gamma / \Omega\left(M_{-1}\right)$ implies that the price and profit flow are given respectively by 


$$
p\left(M_{-1}, M\right)=\left\{\begin{array}{l}
\gamma, M=1, M_{-1}=1, \text { with probability } \theta_{1}^{2} \\
\gamma, M=1, M_{-1} \geq 2, \text { with probability } \theta_{1}\left(1-\theta_{1}\right) \\
\gamma \nu, M \geq 2, M_{-1}=1, \text { with probability } \theta_{1}\left(1-\theta_{1}\right) \\
\gamma, M \geq 2, M_{-1} \geq 2, \text { with probability }\left(1-\theta_{1}\right)^{2}
\end{array}\right.
$$

and

$$
\pi\left(M_{-1}, M\right)=\left\{\begin{array}{l}
\left(\frac{\gamma-1}{\gamma}\right) E, M=1, M_{-1}=1, \text { with probability } \theta_{1}^{2} \\
\left(\frac{\gamma-\nu}{\gamma}\right) E, M=1, M_{-1} \geq 2, \text { with probability } \theta_{1}\left(1-\theta_{1}\right) \\
\left(\frac{\gamma \nu-1}{\gamma \nu}\right) \frac{E}{M}, M \geq 2, M_{-1}=1, \text { with probability } \theta_{1}\left(1-\theta_{1}\right) \\
\left(\frac{\gamma-1}{\gamma}\right) \frac{E}{M}, M \geq 2, M_{-1} \geq 2, \text { with probability }\left(1-\theta_{1}\right)^{2}
\end{array}\right.
$$

Let $V_{C i}\left(k_{-1}\right)$ be the value of $i$ th imitation that occurs in a type $k_{-1}$ sector. ${ }^{28}$ Then we have the Bellman equation

$$
\rho V_{C i}\left(k_{-1}\right)=\left(\frac{\beta \nu-1}{\beta \nu}\right) E /(1+i)-\lambda_{I} n_{I} V_{C i}\left(k_{-1}\right)-\lambda_{C} n_{C}\left[V_{C i}\left(k_{-1}\right)-V_{C(i+1)}\left(k_{-1}\right)\right] .
$$

Following the same calculation procedure as in Appendix 2, we get the value function of the first imitation that occurs in a type $k_{-1}$ sector

$$
V_{C 1}\left(k_{-1}\right)=\left[\psi \ln \left(\frac{\psi}{\psi-1}\right)-1\right] \frac{\left(\frac{\beta \nu-1}{\beta_{\nu}}\right) E}{\lambda_{C} n_{C}}
$$

where $\psi \equiv \frac{\rho+\lambda_{I} n_{I}+\lambda_{C} n_{C}}{\lambda_{C} n_{C}}$. Let $V_{I}\left(k_{-1}\right)$ be the value function of innovation that occurs in a type $k_{-1}$ sector, then we have the Bellman equation

$$
\rho V_{I}\left(k_{-1}\right)=\left(\frac{\beta-1}{\beta}\right) E-\lambda_{I} n_{I} V_{I}\left(k_{-1}\right)-\lambda_{C} n_{C}\left[V_{I}\left(k_{-1}\right)-V_{C 1}\left(k_{-1}\right)\right],
$$

which, along with (87), gives

$$
V_{I}\left(k_{-1}\right)=\left\{\left[\frac{1}{\lambda_{C} n_{C}} \ln \left(1+\frac{\lambda_{C} n_{C}}{\rho+\lambda_{I} n_{I}}\right)\right]\left(\frac{\beta \nu-1}{\beta \nu}\right)-\frac{1}{\rho+\lambda_{I} n_{I}+\lambda_{C} n_{C}}\left(\frac{\nu-1}{\beta \nu}\right)\right\} E .
$$

By assumption, innovations occur randomly and independently across sectors and over time, so the value function of innovation is given by

\footnotetext{
${ }^{28} \mathrm{~A}$ type $k_{-1}$ sector is a sector which is a type $k$ sector in the previous generation.
} 


$$
V_{I}=\sum_{k_{-1}=1}^{\infty} V_{I}\left(k_{-1}\right) \theta_{k_{-1}}=\theta_{1} V_{I}(1)+\left(1-\theta_{1}\right) V_{I}(2)
$$

Substituting $V_{I}(1)$ and $V_{I}(2)$ given by (88) into (89) and rearranging it gives the value function of innovation (35).

To derive the value function $V_{C}$, let $V_{C}\left(k_{-1}\right)$ denote the value of an imitation that occurs in a type $k_{-1}$ sector. Then following exactly the procedure of deriving the value function in the rent-seeking imitation case, we have

$$
\begin{aligned}
& V_{C}\left(k_{-1}\right)=\frac{\lambda_{I} n_{I}}{\rho \lambda_{C} n_{C}}\left[\left(1+\frac{\lambda_{I} n_{I}}{\lambda_{C} n_{C}}\right) \ln \left(1+\frac{\lambda_{C} n_{C}}{\lambda_{I} n_{I}}\right)\right. \\
&\left.-\left(1+\frac{\rho+\lambda_{I} n_{I}}{\lambda_{C} n_{C}}\right) \ln \left(1+\frac{\lambda_{C} n_{C}}{\rho+\lambda_{I} n_{I}}\right)\right]\left(\frac{\beta \nu-1}{\beta \nu}\right) E .
\end{aligned}
$$

Since it is assumed that imitations also occur randomly and independently across sectors and over time, we get the value function of imitation by taking the expectation of $V_{C}\left(k_{-1}\right)$. That is,

$$
V_{C}=\sum_{k_{-1}=1}^{\infty} V_{C}\left(k_{-1}\right) \theta_{k_{-1}}=\theta_{1} V_{C}(1)+\left(1-\theta_{1}\right) V_{C}(2)
$$

which, together with (90), gives (36). Notice that if $\nu=1$, then (35) and (36) are respectively the same as (19) and (21) in section 2.

\section{Appendix 13: Derivation of Equation (37)}

From (85) and (86) in Appendix 12, we have

$$
\begin{aligned}
X & =\sum_{k=1}^{\infty}\left[\sum_{k=1}^{\infty}\left(\frac{E}{p}\right) \theta_{k}\right] \theta_{k_{-1}} \\
& =\sum_{k_{-1}=1}^{\infty}\left[\theta_{1} \frac{1}{\beta}+\left(1-\theta_{1}\right) \frac{1}{\beta \nu}\right] \theta_{k_{-1}} E \\
& =\left\{\theta_{1}\left[\theta_{1} \frac{1}{\gamma}+\left(1-\theta_{1}\right) \frac{\nu}{\gamma}\right]+\left(1-\theta_{1}\right)\left[\theta_{1} \frac{1}{\gamma \nu}+\left(1-\theta_{1}\right) \frac{1}{\gamma}\right]\right\} E
\end{aligned}
$$

Rearranging (92) gives (37). 
Figure 1: The Value of Innovation

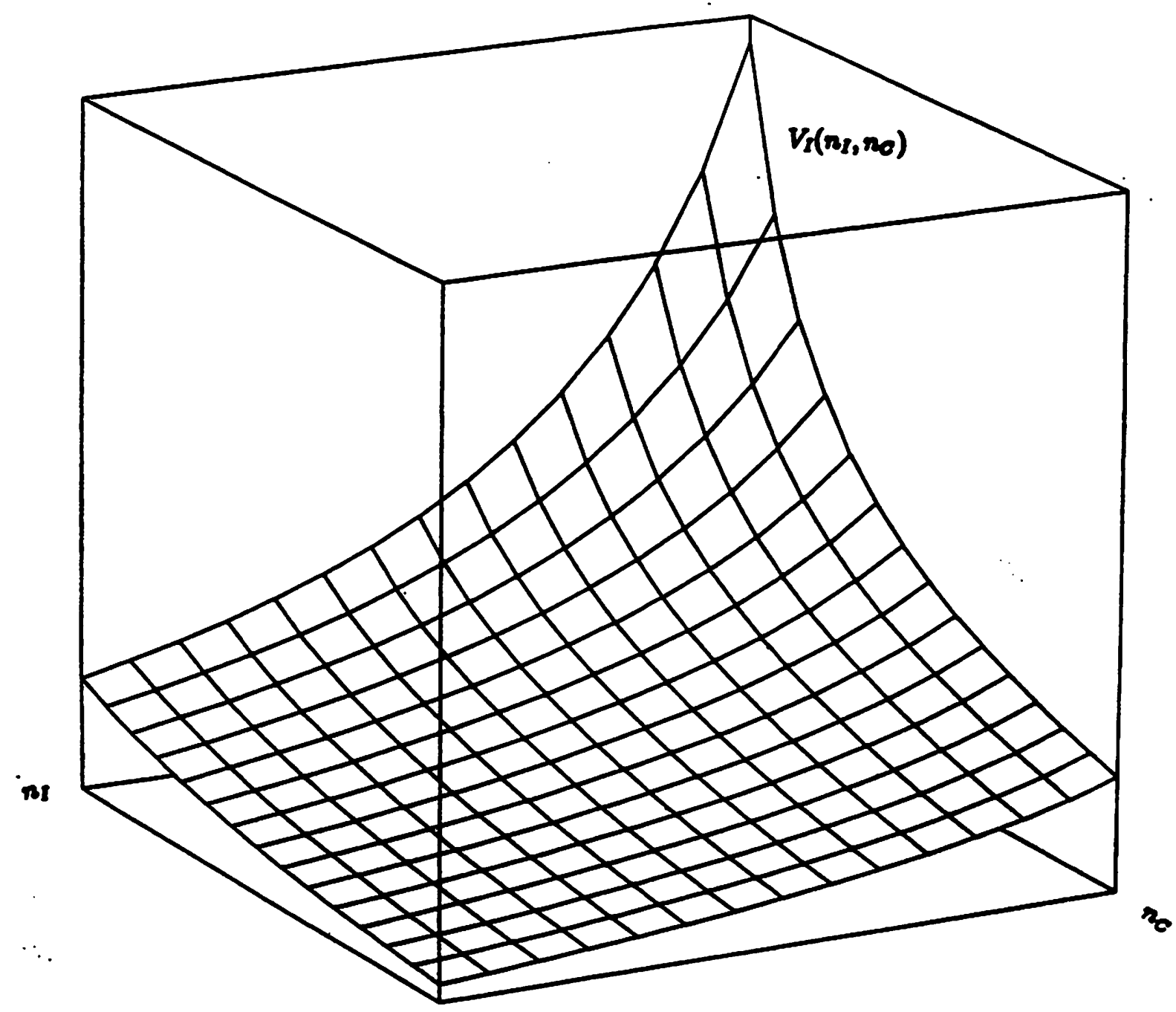


Figure 2: The Value of Imitation

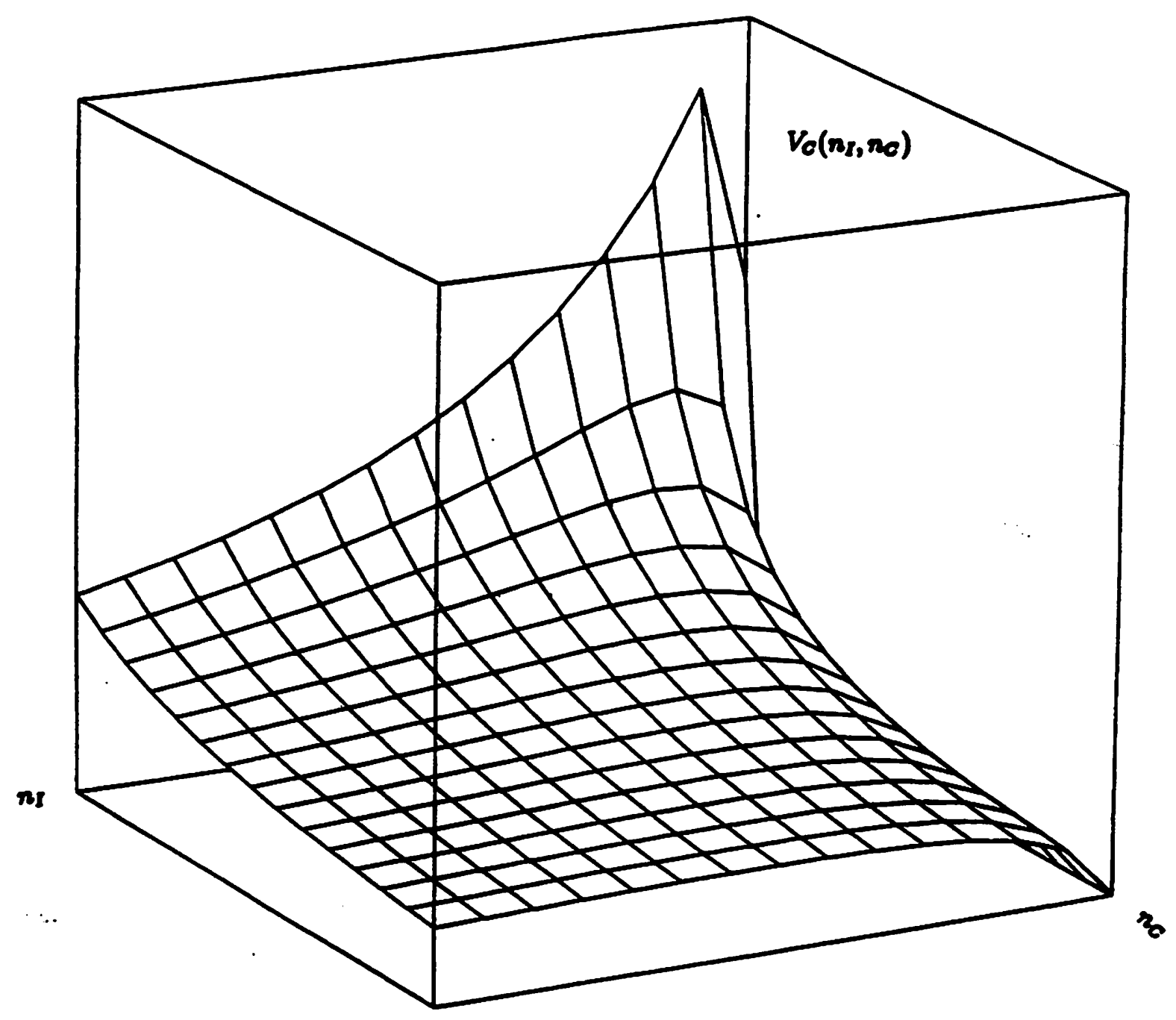




\section{References}

[1] Aghion, Phillippe and Peter Howitt, A Model of Growth Through Creative Destruction, Unpublished, the University of Western Ontario, 1991.

[2] - A Model of Growth Through Creative Destruction, Econometrica 60 (March 1992): 323-351.

[3] - Research and Development in the Growth Process, Unpublished, the University of Western Ontario, 1994.

[4] Baumol, William J., Entrepreneurship: Productive, Unproductive, and Destructive, Journal of Political Economy XCVIII (1990): 893-921.

[5] Dasgupta, Partha and Joseph Stiglitz, Uncertainty, Industry Structure and the Speed of R\&D, Bell Journal of Economics 11 (Spring 1980): 1-28.

[6] Davidson, Carl and Paul Segerstrom, Patent Enforcement and Economic Growth, Michigan State University Working Paper, 1993.

[7] Dinopoulos, Elias, Schumpeterian Product Evolution and Vanishing Growth, University of Florida Working Paper, 1991.

[8] Grossman, Gene and Elhanan Helpman, Quality Ladders in the Theory of Growth, Review of Economic Studies 58 (January 1991a): 43-61.

[9] —, Quality Ladder and Product Cycles, Quarterly Journal of Economics 106 (1991b): $557-586$.

[10] -, Innovation and Growth in the Global Economy, Cambridge: MIT Press, 1991c.

[11] Jovanovic, Boyan and Rafael Rob, Long Waves and Short Waves: Growth through Intensive and Extensive Search, Econometrica 58 (November 1990): 1391-1409. 
[12] Lee, Tom and Louis Wide, Market Structure and Innovation: A Reformulation, Quarterly Journal of Economics 94 (March 1980): 429436.

[13] Loury, Glen, Market Structure and Innovation, Quarterly Journal of Economics 93 (August 1979): 395-410.

[14] Lucas, Robert Jr., On the Mechanics of Economic Development, Journal of Monetary Economics 22 (1988):3-42.

[15] Mansfield, Edwin, Mark Schwartz and Samuel Wagner, Imitation Costs and Patents: An Empirical Study, Economic Journal 91 (December 1981): 907-18.

[16] Mitrinovic, D.S., Analytic Inequalities, Berlin, New York: Springer-Verlag, 1970.

[17] Murphy, Kevin M, Andrei Shleifer and Robert W. Vishny, The Allocation of Talent: Implications for Growth, Quarterly Journal of Economics (May 1991): 503-530.

[18] Rebelo, Sergio, Long-Run Policy Analysis and Long-Run Growth, Journal of Political Economy 99 (1991): 500-21.

[19] Reinganum, Jannifer, The Timing of Innovation: Research, Development and Diffusion, In Handbook of Industrial Organization, Vol. I, ed. by R. Schmalensee and R. Willig, Amsterdam: North-Holland, 1989.

[20] Romer, Paul, Increasing Returns and Long-Run Growth, Journal of Political Economy 94 (1986): 1002-1037.

[21] —_, Endogenous Technological Change, Journal of Political Economy 98 (1990): S71S102.

[22] — Two Strategies for Economic Development: Using Ideas and Producing Ideas, Working paper (March 1992). 
[23] Schmitz, James A., Jr., Imitation, Entrepreneurship, and Long-Run Growth, Journal of Political Economy 97 (1989): 721-739.

[24] Segerstrom, Paul, Innovation, Imitation and Economic Growth, Journal of Political Economy 99 (August 1991): 807-827.

[25] Segerstrom, Paul, T.C.A. Anant, and Elias Dinopoulos, A Schumpeterian Model of the Product Life Cycle, American Economic Review 80 (December 1990):1077-91.

[26] Stokey, Nancy, Learning by Doing and the Introduction of New Goods, Journal of Political Economy 96 (1988): 701-717.

[27] —, Human Capital, Product Quality, and Growth, Quarterly Journal of Economics CVI (May 1991): 587-616.

[28] Young, Alwyn, Invention and Bounded Learning by Doing, NBER working paper No. 3712 (May 1991). 